\title{
EXPONENTIAL INTEGRATORS FOR STOCHASTIC SCHRÖDINGER EQUATIONS DRIVEN BY ITO NOISE
}

\author{
RIKARD ANTON* AND DAVID COHEN ${ }^{\dagger}$
}

\begin{abstract}
We study an explicit exponential scheme for the time discretisation of stochastic Schrödinger equations driven by additive or multiplicative Ito noise. The numerical scheme is shown to converge with strong order 1 if the noise is additive and with strong order $1 / 2$ for multiplicative noise. In addition, if the noise is additive, we show that the exact solutions of our problems satisfy trace formulas for the expected mass, energy, and momentum (i. e., linear drifts in these quantities). Furthermore, we inspect the behaviour of the numerical solutions with respect to these trace formulas. Several numerical simulations are presented and confirm our theoretical results.
\end{abstract}

Key words. Stochastic partial differential equations; Stochastic Schrödinger equations; Numerical methods; Geometric numerical integration; Stochastic exponential integrators; Strong convergence; Trace formulas

AMS subject classifications. 35Q55, 60H15, 65C20, 65C30, 65C50, 65J08

1. Introduction. We consider temporal discretisations of nonlinear stochastic Schrödinger equations driven by Ito noise

$$
\begin{array}{ll}
\operatorname{id} u=\Delta u \mathrm{~d} t+F(x, u) \mathrm{d} t+G(u) \mathrm{d} W & \text { in } \mathbb{R}^{d} \times(0, \infty), \\
u(\cdot, 0)=u_{0} & \text { in } \mathbb{R}^{d},
\end{array}
$$

where $u=u(x, t)$, and $\mathrm{i}=\sqrt{-1}$. The product between $G$ and $\mathrm{d} W$ is of Ito type, and further details on $F$ and $G$ and on the dimension $d$ will be specified later. The stochastic process $\{W(t)\}_{t \geq 0}$ is a square integrable complex-valued $Q$-Wiener process with respect to a normal filtration $\left\{\mathscr{F}_{t}\right\}_{t \geq 0}$ on a filtered probability space $\left(\Omega, \mathscr{F}, \mathbb{P},\left\{\mathscr{F}_{t}\right\}_{t \geq 0}\right)$. The regularity of the covariance operator $Q$ will be specified later in the text. The initial value $u_{0}$ is an $\mathscr{F}_{0}$-measurable complex-valued function, which will be further specified below.

The Schrödinger equation is widely used within physics and takes several different forms depending on the situation. It is used in hydrodynamics, nonlinear optics and plasma physics to only mention a few areas. In certain physical situations it may be appropriate to incorporate some kind of randomness into the equation. One possibility is to add a driving random force to then obtain an equation of the form (1.1). See for example [12] and references therein for further details.

Stochastic Schrödinger equations have received much attention from a more theoretical point of view during the last decades. Connected to the present article and without being exhaustive, we mention the works [13, 14, 18] on Ito problems and [12, 14, 16, 30, 18] for the Stratonovich setting.

It is seldom possible to solve stochastic partial differential equations exactly, and efficient numerical schemes are therefore needed. For the time integration of the above stochastic Schrödinger equations, we will consider stochastic exponential integrators. These numerical methods are explicit and easy to implement, furthermore they offer good geometric properties. Exponential integrators are widely used and studied nowadays as witnessed by the recent review [23] for the time integration of deterministic problems. Applications of such schemes to the deterministic (nonlinear) Schrödinger equation can be found in, for example,

${ }^{*}$ Department of Mathematics and Mathematical Statistics, Umeå University, SE-901 87 Umeå, Sweden (rikard.anton@umu.se).

${ }^{\dagger}$ Department of Mathematics and Mathematical Statistics, Umeå University, SE-901 87 Umeå, Sweden (david.cohen@umu.se). Department of Mathematics, University of Innsbruck, A-6020 Innsbruck, Austria (david.cohen@uibk.ac.at) 
$[22,4,3,7,20,8,5]$ and references therein. These numerical methods were recently investigated for stochastic parabolic partial differential equations in, for example, [28, 24, 29] and for the stochastic wave equations in [9, 32, 10, 1].

We now review previous works on temporal discretisations of stochastic Schrödinger equations. In [17] a Crank-Nicolson scheme is studied for the equation with nonlinearity $F(u)$. First order of convergence is obtained in the case of additive noise, and with multiplicative Ito noise the convergence rate is one half. Observe that this numerical scheme is implicit. A stochastic Schrödinger equation with Stratonovich noise is considered in [15], where, again, a Crank-Nicolson scheme is studied for the equation with nonlinearity $F(x, u)=\lambda|u|^{2 \sigma} u$, with $\lambda= \pm 1$ and $\sigma>0$. The authors prove convergence to the exact solution and mass preservation of the scheme. Further, in [26] a mass-preserving splitting scheme for equation (1.1) with $F(x, u)=V(x) u$ and $G(u)=u$ is considered. The noise is of Stratonovich type and first order convergence is obtained. In [27], $V(x)$ is replaced by $|u|^{2}$ and first order convergence is again obtained. Still in the Stratonovich setting, [25] derives multi-symplectic schemes for stochastic Schrödinger equations. We finally mention [19, 2], in which thorough numerical simulations are presented for both additive noise and multiplicative Stratonovich noise.

In the present work we show that

- the exponential integrator applied to the linear stochastic Schrödinger equation with additive noise converges strongly with order 1 and satisfies exact trace formulas for the mass, the energy, and for the momentum;

- the exponential integrator applied to the stochastic Schrödinger equation with a multiplicative potential and additive noise converges with strong order 1, but has a small error in the trace formulas for the mass and energy;

- the exponential integrator applied to stochastic Schrödinger equations driven by multiplicative Ito noise strongly converges with order $1 / 2$.

We begin the exposition by introducing some notations and useful results that we will use in our proofs. After that we will follow a similar approach as in [17]. That is, we will begin by analysing the numerical method applied to the linear Schrödinger equation with additive noise in Section 3 Then we study stochastic Schrödinger equations with a multiplicative potential in Section 14 and finally we consider the stochastic Schrödinger equation with a multiplicative potential and multiplicative noise in Section 5. For each of the above problems, we analyse the speed of convergence of the exponential methods (in the strong sense) and for additive problems we show some trace formulas (such results could be interpreted as weak error estimates). Various numerical experiments accompany the presentation and illustrate the main properties of these exponential methods when applied to stochastic Schrödinger equations driven by Ito noise.

2. Notations and some useful results. Given two separable Hilbert spaces $H_{1}$ and $H_{2}$ with norms $\|\cdot\|_{H_{1}}$ and $\|\cdot\|_{H_{2}}$ respectively, we denote the space of bounded linear operators from $H_{1}$ to $H_{2}$ by $\mathscr{L}\left(H_{1}, H_{2}\right)$. We denote by $\mathscr{L}_{2}\left(H_{1}, H_{2}\right)$ the set of Hilbert-Schmidt operators from $H_{1}$ to $H_{2}$ with norm

$$
\|\Phi\|_{\mathscr{L}_{2}\left(H_{1}, H_{2}\right)}:=\left(\sum_{k=1}^{\infty}\left\|\Phi e_{k}\right\|_{H_{2}}^{2}\right)^{1 / 2},
$$

where $\left\{e_{k}\right\}_{k=1}^{\infty}$ is any orthonormal basis of $H_{1}$. Furthermore, for Hilbert spaces $H_{1}, H_{2}$, and $H_{3}$ we have (see the proof of Lemma 2.1 in [12]) that if $S \in \mathscr{L}_{2}\left(H_{1}, H_{2}\right)$ and $T \in \mathscr{L}\left(H_{2}, H_{3}\right)$, then $T S \in \mathscr{L}_{2}\left(H_{1}, H_{3}\right)$ and

$$
\|T S\|_{\mathscr{L}_{2}\left(H_{1}, H_{3}\right)} \leq\|T\|_{\mathscr{L}\left(H_{2}, H_{3}\right)}\|S\|_{\mathscr{L}_{2}\left(H_{1}, H_{2}\right)} .
$$


Also, we consider $L^{2}\left(\mathbb{R}^{d}\right)$ the space of square integrable functions on $\mathbb{R}^{d}$ with inner product

$$
(u, v)=\operatorname{Re} \int_{\mathbb{R}^{d}} u \bar{v} \mathrm{~d} x .
$$

For $\sigma \in \mathbb{R}$, we further denote the fractional Sobolev space of order $\sigma$ by $H^{\sigma}=H^{\sigma}\left(\mathbb{R}^{d}\right)$ with norm $\|\cdot\|_{\sigma}$. To make the notations cleaner and more readable we will use the following shorter notations

$$
L^{2}=L^{2}\left(\mathbb{R}^{d}\right), \quad \mathscr{L}\left(H_{1}\right)=\mathscr{L}\left(H_{1}, H_{1}\right), \quad \mathscr{L}_{2}^{\sigma}=\mathscr{L}_{2}\left(L^{2}, H^{\sigma}\right) .
$$

We note that the operator $-\mathrm{i} \Delta$, appearing in the stochastic Schrödinger equation, is the generator of a semigroup of isometries of bounded linear operators $S(t)=\mathrm{e}^{-\mathrm{i} t \Delta}$. We will make use of the following result

Lemma 2.1. (See e.g. [26] Lemma 3.2]) Consider any $\sigma \geq 0$ and $S(t)=\mathrm{e}^{-\mathrm{i} t \Delta}, t \geq 0$, then, for $w \in H^{\sigma}$, one has

$$
\|S(t) w\|_{\sigma}=\|w\|_{\sigma}
$$

and, for $\Delta w \in H^{\sigma}$ and any $t \geq 0$,

$$
\|\Delta(S(t) w)\|_{\sigma}=\|\Delta w\|_{\sigma}, \quad\|(S(t)-I) w\|_{\sigma} \leq t\|\Delta w\|_{\sigma} .
$$

As a consequence of the above lemma and $\left[2.1\right.$, we have, for $\Phi \in \mathscr{L}_{2}^{\sigma+2}$ and any $t \geq 0$,

$$
\|(S(t)-I) \Phi\|_{\mathscr{L}_{2}^{\sigma}} \leq t\|\Phi\|_{\mathscr{L}_{2}^{\sigma+2}}
$$

We will also use the following result.

LEMMA 2.2. For any $\sigma \geq 0$ and $t \geq 0$, we have

$$
\|S(t)-I\|_{\mathscr{L}\left(H^{\sigma+1}, H^{\sigma}\right)} \leq C t^{1 / 2},
$$

for a constant $C$.

The proof of this result is very similar to the proof of Lemma 2.1.

Finally, throughout the paper, $C$ (and $C_{1}, C_{2}$ etc.) will denote a generic constant which may change from line to line.

3. The linear Schrödinger equation with additive noise. In this section, we study time discretisations of the linear stochastic Schrödinger equation

$$
\begin{array}{ll}
\mathrm{id} u-\Delta u \mathrm{~d} t=\mathrm{d} W & \text { in } \mathbb{R}^{d} \times(0, \infty), \\
u(0)=u_{0} & \text { in } \mathbb{R}^{d},
\end{array}
$$

where $u_{0}$ is an $\mathscr{F}_{0}$-measurable random variable and the noise $\{W(t)\}_{t \geq 0}$ is a square integrable complex-valued $Q$-Wiener process with respect to the filtration. In this section, we have no restrictions on the dimension $d$.

Global existence and uniqueness in $H^{\sigma}, \sigma \geq 0$, of the solution to equation (3.1) is guaranteed if $u_{0} \in H^{\sigma}$ a.s. and $Q^{1 / 2} \in \mathscr{L}_{2}^{\sigma}$. The proof follows the same line as the proof of Theorem 7.4 in [11]. The mild solution of (3.1) reads

$$
u(t)=S(t) u_{0}-\mathrm{i} \int_{0}^{t} S(t-r) \mathrm{d} W(r),
$$

where we recall the notation $S(t)=\mathrm{e}^{-\mathrm{i} t \Delta}$. 
We now consider the time integration of the above problem. This is done as follows. Let $T>0$ be a fixed time horizon and $N>0$ be an integer. We first divide the interval $[0, T]$ into subintervals $0=t_{0}<t_{1}<\ldots<t_{N-1}<t_{N}=T$ of equal length $k$ so that $t_{n}=n k$. An exponential integrator with step size $k$ is now derived by approximating the above stochastic integral, in the mild solution, at the left end point. We thus obtain a numerical approximation $u^{n}$ of the exact solution $u\left(t_{n}\right)$ of 3.1 :

$$
u^{n}=S(k) u^{n-1}-\mathrm{i} S(k) \Delta W^{n-1}=S\left(t_{n}\right) u_{0}-\mathrm{i} \sum_{j=0}^{n-1} \int_{t_{j}}^{t_{j+1}} S\left(t_{n}-t_{j}\right) \mathrm{d} W(r),
$$

where $\Delta W^{n-1}=W^{n}-W^{n-1}=W\left(t_{n}\right)-W\left(t_{n-1}\right)$ denotes Wiener increments. We call this explicit numerical method an exponential integrator.

3.1. Error estimates. This subsection presents a result on the error of the exponential integrator (3.2) when applied to the linear stochastic Schrödinger equation 3.1]. These error estimates are given in the next theorem.

THEOREM 3.1. Let $\sigma \geq 0, p \in \mathbb{N}$. Recall that $u^{n}$ is the numerical approximation given by the exponential integrator (3.2) of the exact solution $u\left(t_{n}\right)$ to the linear stochastic Schrödinger equation driven by a $Q$-Wiener process (3.1). Assume that $u_{0} \in H^{\sigma}$ a.s., and $Q^{1 / 2} \in \mathscr{L}_{2}^{\sigma+2}$. Then there exists a constant $C$ such that

$$
\mathbb{E}\left[\max _{n=1, \ldots, N}\left\|u^{n}-u\left(t_{n}\right)\right\|_{\sigma}^{2 p}\right] \leq C k^{2 p}\left\|Q^{1 / 2}\right\|_{\mathscr{L}_{2}^{\sigma+2}}^{2 p} .
$$

Proof. We have

$$
\begin{aligned}
u^{n}-u\left(t_{n}\right) & =\mathrm{i} \sum_{j=0}^{n-1} \int_{t_{j}}^{t_{j+1}}\left(S\left(t_{n}-r\right)-S\left(t_{n}-t_{j}\right)\right) \mathrm{d} W(r) \\
& =\mathrm{i} \int_{0}^{t_{n}}\left(S\left(t_{n}-r\right)-S\left(t_{n}-[r / k] k\right)\right) \mathrm{d} W(r),
\end{aligned}
$$

where $[r / k]$ denotes the integer part of $r / k$. The last equality is indeed correct since if $t_{j} \leq$ $r<t_{j+1}$, then $j \leq r / k<j+1$ so that $[r / k]=j$ and $[r / k] k=j k=t_{j}$. Using Burkholder's inequality [11, Lemma 7.2], and Lemma2.1] we have

$$
\begin{aligned}
\mathbb{E}\left[\max _{n=1, \ldots, N}\left\|u^{n}-u\left(t_{n}\right)\right\|_{\sigma}^{2 p}\right] & \leq \mathbb{E}\left[\sup _{t \in[0, T]}\left\|\mathrm{i} \int_{0}^{t}(S(t-r)-S(t-[r / k] k)) \mathrm{d} W(r)\right\|_{\sigma}^{2 p}\right] \\
& \leq C \mathbb{E}\left[\left(\int_{0}^{T}\left\|(S([r / k] k-r)-I) Q^{1 / 2}\right\|_{\mathscr{L}_{2}^{\sigma}}^{2} \mathrm{~d} r\right)^{p}\right] \\
& =C \mathbb{E}\left[\left(\sum_{j=0}^{N-1} \int_{t_{j}}^{t_{j+1}}\left\|\left(S\left(t_{j}-r\right)-I\right) Q^{1 / 2}\right\|_{\mathscr{L}_{2}^{\sigma}}^{2} \mathrm{~d} r\right)^{p}\right] \\
& \leq C \mathbb{E}\left[\left(\sum_{j=0}^{N-1} \int_{t_{j}}^{t_{j+1}}\left|r-t_{j}\right|^{2}\left\|Q^{1 / 2}\right\|_{\mathscr{L}_{2}^{\sigma+2}}^{2} \mathrm{~d} r\right)^{p}\right] \\
& \leq C k^{2 p}\left\|Q^{1 / 2}\right\|_{\mathscr{L}_{2}^{\sigma+2}}^{2 p} .
\end{aligned}
$$


3.2. A trace formula for the mass. Under appropriate boundary conditions, for example periodic boundary conditions or homogeneous Dirichlet boundary conditions, the mass (also called $L^{2}$-norm or density)

$$
M(u):=\int|u|^{2} \mathrm{~d} x
$$

of the deterministic linear Schrödinger equation $\mathrm{i} \frac{\partial u}{\partial t}-\Delta u=0$ is a conserved quantity. In the stochastic case, one immediately gets a trace formula for the mass of the exact solution as stated below.

Proposition 3.2. Assume that the initial data is such that $\mathbb{E}\left[M\left(u_{0}\right)\right]$ is finite and that the covariance operator $Q$ is trace-class. Then the exact solution of the linear Schrödinger equation with additive noise (3.1) satisfies the trace formula for the expected mass

$$
\mathbb{E}[M(u(t))]=\mathbb{E}\left[\|u(t)\|_{L^{2}}^{2}\right]=\mathbb{E}\left[M\left(u_{0}\right)\right]+t \operatorname{Tr}(Q) \quad \text { for all time } t .
$$

Proof. Using Lemma2.1 and the fact that the stochastic integrals are normally distributed with mean 0 , we get

$$
\begin{aligned}
\mathbb{E}\left[\|u(t)\|_{L^{2}}^{2}\right]= & \mathbb{E}\left[\left\|S(t) u_{0}\right\|_{L^{2}}^{2}+\left(S(t) u_{0},-\mathrm{i} \int_{0}^{t} S(t-r) \mathrm{d} W(r)\right)\right. \\
& \left.+\left(-\mathrm{i} \int_{0}^{t} S(t-r) \mathrm{d} W(r), S(t) u_{0}\right)+\left\|\int_{0}^{t} S(t-r) \mathrm{d} W(r)\right\|_{L^{2}}^{2}\right] \\
= & \mathbb{E}\left[\left\|S(t) u_{0}\right\|_{L^{2}}^{2}\right]+\int_{0}^{t} \mathbb{E}\left[\left\|S(t-r) Q^{1 / 2}\right\|_{\mathscr{L}_{2}^{0}}^{2} \mathrm{~d} r\right. \\
= & \mathbb{E}\left[\left\|u_{0}\right\|_{L^{2}}^{2}\right]+t \operatorname{Tr}(Q) .
\end{aligned}
$$

REMARK 3.3. We would like to point out that, as in the case of the linear stochastic wave equation treated in [9], an alternative proof of the above result can be obtained using Ito's formula (see, for example [11 Theorem 4.17]). This remark is also valid for the other trace formulas given below.

Our exponential integrator does indeed satisfy this trace formula for the mass as well, as seen in the next result.

PROPOSITION 3.4. With the same assumptions as in Proposition 3.2 the stochastic exponential integrator (3.2) satisfies the following trace formula for the mass

$$
\begin{aligned}
\mathbb{E}\left[M\left(u^{n}\right)\right] & =\mathbb{E}\left[M\left(u^{n-1}\right)\right]+k \operatorname{Tr}(Q) \\
& =\mathbb{E}\left[M\left(u_{0}\right)\right]+t_{n} \operatorname{Tr}(Q) \text { for all } t_{n}=n k
\end{aligned}
$$

Proof. Similarly to the proof of Proposition 3.2, we have

$$
\begin{aligned}
\mathbb{E}\left[M\left(u^{n}\right)\right] & =\mathbb{E}\left[M\left(u^{n-1}\right)\right]+\mathbb{E}\left[\left\|\int_{t_{n-1}}^{t_{n}} S(k) \mathrm{d} W(r)\right\|_{L^{2}}^{2}\right] \\
& =\mathbb{E}\left[M\left(u^{n-1}\right)\right]+k \operatorname{Tr}(Q) .
\end{aligned}
$$

A recursion concludes the proof.

We would like to examine the behaviour of the Euler-Maruyama scheme, the backward Euler-Maruyama scheme, and the midpoint rule with respect to the trace formula for the mass. 
Since we will consider periodic domains and pseudospectral discretisations in the numerical experiments presented below, we will examine this case first.

A pseudospectral spatial discretisation of 3.1 with a noise given by the representation

$$
W(x, t)=\sum_{n \in \mathbb{Z}} \lambda_{n}^{1 / 2} \beta_{n}(t) e_{n}(x),
$$

where $\left\{\beta_{n}(t)\right\}_{n \in \mathbb{Z}}$ are i.i.d Brownian motions, $\lambda_{n}$ are eigenvalues of $Q$, and $\left\{e_{n}(x)\right\}_{n \in \mathbb{Z}}=$ $\left\{\frac{1}{\sqrt{2 \pi}} \mathrm{e}^{\mathrm{i} n x}\right\}_{n \in \mathbb{Z}}$ is an orthonormal basis of $L^{2}(0,2 \pi)$, will lead to the following system of decoupled stochastic differential equations for the Fourier coefficients $y_{k}$ of the exact solution

$$
\operatorname{id} y_{k}=-k^{2} y_{k} \mathrm{~d} t+\lambda_{k}^{1 / 2} \mathrm{~d} \beta_{k} .
$$

This motivates us to consider the scalar test problem (with a standard Brownian motion $\beta$ and real numbers $a, b$ )

$$
\operatorname{id} y=a y \mathrm{~d} t+b \mathrm{~d} \beta
$$

and the quantity equivalent to the mass is thus the second moment $\mathbb{E}\left[|y|^{2}\right]$. We thus have the trace formula for the exact solution

$$
\mathbb{E}\left[|y(t)|^{2}\right]=\mathbb{E}\left[|y(0)|^{2}\right]+b^{2} t \quad \text { for all times } \quad t .
$$

The following result states that the above classical numerical methods do not preserve the trace formula for this simple test problem, and in particular, are not suited when applied to pseudospectral discretisations of linear stochastic Schrödinger equations.

PROPOSITION 3.5. Consider a pseudospectral discretisation of the stochastic Schrödinger equation (3.1) with a trace-class noise and periodic boundary conditions yielding to equations of the form (3.3). We have the following results:

\section{The Euler-Maruyama scheme}

$$
y^{n+1}=y^{n}-\mathrm{i} k a y^{n}-\mathrm{i} b \Delta W^{n}
$$

produces a second moment that grows exponentially with time

$$
\mathbb{E}\left[\left|y^{n}\right|^{2}\right] \geq \mathrm{e}^{\left(\frac{1}{2} k a^{2}\right) t_{n}} \mathbb{E}\left[\left|y^{0}\right|^{2}\right] \quad \text { for } \quad t_{n}=n k .
$$

2. The backward Euler-Maruyama scheme

$$
y^{n+1}=y^{n}-\mathrm{i} k a y^{n+1}-\mathrm{i} b \Delta W^{n}
$$

produces a second moment that grows at a slower rate than the exact solution

$$
\mathbb{E}\left[\left|y^{n}\right|^{2}\right] \leq \mathbb{E}\left[\left|y^{0}\right|^{2}\right]+\frac{b^{2}}{a^{2} k} \quad \text { for all } n \geq 0,
$$

hence $\lim _{t_{n} \rightarrow \infty}\left(\mathbb{E}\left[\left|y^{n}\right|^{2}\right] / t_{n}\right)=0$.

3. The midpoint rule

$$
\mathrm{i} \frac{y^{n+1}-y^{n}}{k}-a \frac{y^{n+1}+y^{n}}{2}=b \Delta W^{n}
$$

produces a second moment that underestimate the linear drift of the exact solution

$$
\mathbb{E}\left[\left|y^{n}\right|^{2}\right]=\mathbb{E}\left[\left|y^{0}\right|^{2}\right]+\frac{b^{2} t_{n}}{1+\frac{a^{2} k^{2}}{2}} \quad \text { for } \quad t_{n}=n k
$$


Proof. The proof of this proposition is an easy adaptation of the results presented in [31]. We start by looking at the behaviour of the Euler-Maruyama scheme and compute, using properties of the Wiener increments,

$$
\begin{aligned}
\mathbb{E}\left[\left|y^{n+1}\right|^{2}\right] & =\left(1+a^{2} k^{2}\right) \mathbb{E}\left[\left|y^{n}\right|^{2}\right]+b^{2} k \geq\left(1+a^{2} k^{2}\right) \mathbb{E}\left[\left|y^{n}\right|^{2}\right] \geq\left(1+a^{2} k^{2}\right)^{n+1} \mathbb{E}\left[\left|y^{0}\right|^{2}\right] \\
& \geq \mathrm{e}^{\left(\frac{1}{2} k a^{2}\right) t_{n+1}} \mathbb{E}\left[\left|y^{0}\right|^{2}\right]
\end{aligned}
$$

For the backward Euler-Maruyama scheme we obtain, using a geometric series,

$$
\mathbb{E}\left[\left|y^{n+1}\right|^{2}\right]=\frac{1}{1+a^{2} k^{2}}\left(\mathbb{E}\left[\left|y^{n}\right|^{2}\right]+b^{2} k\right) \leq \mathbb{E}\left[\left|y^{n}\right|^{2}\right]+\frac{b^{2} k}{1+a^{2} k^{2}} \leq \mathbb{E}\left[\left|y^{0}\right|^{2}\right]+\frac{b^{2}}{a^{2} k}
$$

Finally, for the midpoint rule, we have

$$
\mathbb{E}\left[\left|y^{n+1}\right|^{2}\right]=\mathbb{E}\left[\left|y^{n}\right|^{2}\right]+\frac{b^{2} k}{1+\frac{a^{2} k^{2}}{2}}=\mathbb{E}\left[\left|y^{0}\right|^{2}\right]+\frac{b^{2} t_{n+1}}{1+\frac{a^{2} k^{2}}{2}}
$$

For homogeneous Dirichlet boundary conditions a similar result holds.

PROPOSITION 3.6. Consider the stochastic Schrödinger equation 3.1 with a traceclass noise and homogeneous Dirichlet boundary conditions. We have the following results:

1. The Euler-Maruyama scheme

$$
u^{n+1}=u^{n}-\mathrm{i} k \Delta u^{n}-\mathrm{i} \Delta W^{n}
$$

produces a numerical trace formula for the mass that grows exponentially with time

$$
\mathbb{E}\left[M\left(u^{n}\right)\right] \geq \mathrm{e}^{\left(\frac{1}{2} k \lambda_{1}\right) t_{n}} \mathbb{E}\left[M\left(u^{0}\right)\right] \quad \text { for } \quad t_{n}=n k,
$$

where $\lambda_{1}$ denotes the smallest eigenvalue of the Laplacian.

2. The backward Euler-Maruyama scheme

$$
u^{n+1}=u^{n}-\mathrm{i} k \Delta u^{n+1}-\mathrm{i} \Delta W^{n}
$$

produces a numerical trace formula for the mass that grows at a slower rate than the exact solution

$$
\mathbb{E}\left[M\left(u^{n}\right)\right] \leq \mathbb{E}\left[M\left(u^{0}\right)\right]+\frac{\operatorname{Tr}(Q)}{\lambda_{1}^{2} k}, \text { for all } n \geq 0,
$$

where $\lambda_{1}$ is the smallest eigenvalue of the Laplacian. Thus $\lim _{t_{n} \rightarrow \infty}\left(\mathbb{E}\left[M\left(u^{n}\right)\right] / t_{n}\right)=0$.

3. The midpoint rule [17]

$$
\mathrm{i} \frac{u^{n+1}-u^{n}}{k}-\Delta \frac{u^{n+1}+u^{n}}{2}=\Delta W^{n}
$$

underestimate the expected mass:

$$
\mathbb{E}\left[M\left(u^{n}\right)\right] \leq \mathbb{E}\left[M\left(u^{0}\right)\right]+\frac{t_{n}}{1+\frac{k^{2} \lambda_{1}}{4}} \operatorname{Tr}(Q), \quad \text { for all } \quad n \geq 0
$$

where $\lambda_{1}$ is the smallest eigenvalue of the Laplacian.

Proof. 
1. We start by looking at the behaviour of the Euler-Maruyama scheme and compute

$$
\mathbb{E}\left[M\left(u^{n}\right)\right]=\mathbb{E}\left[M\left(u^{n-1}\right)\right]+k^{2} \mathbb{E}\left[\left\|\Delta u^{n-1}\right\|^{2}\right]+k \operatorname{Tr}(Q) \geq \mathbb{E}\left[M\left(u^{n-1}\right)\right]+k^{2} \lambda_{1}^{2} \mathbb{E}\left[\left\|u^{n-1}\right\|^{2}\right]+k \operatorname{Tr}(Q),
$$

where $\lambda_{1}$ denotes the smallest eigenvalue of the Laplacian. Hence, one obtains

$$
\mathbb{E}\left[M\left(u^{n}\right)\right] \geq\left(1+k^{2} \lambda_{1}^{2}\right) \mathbb{E}\left[M\left(u^{n-1}\right)\right] \geq\left(1+k^{2} \lambda_{1}^{2}\right)^{n} \mathbb{E}\left[M\left(u^{0}\right)\right] \geq \mathrm{e}^{\left(\frac{1}{2} k \lambda_{1}\right) t_{n}} \mathbb{E}\left[M\left(u^{0}\right)\right] .
$$

2. For the backward Euler-Maruyama scheme we obtain

$$
u^{n+1}+\mathrm{i} k \Delta u^{n+1}=u^{n}-\mathrm{i} \Delta W^{n} .
$$

Taking the norm and expectation we have

$$
\mathbb{E}\left[\left\|u^{n+1}\right\|^{2}\right]+k^{2} \mathbb{E}\left[\left\|\Delta u^{n+1}\right\|^{2}\right]=\mathbb{E}\left[\left\|u^{n}\right\|^{2}\right]+\mathbb{E}\left[\left\|\Delta W^{n}\right\|^{2}\right] .
$$

Using that the eigenvalues are positive and increasing, and using a similar argument as in the proof of Proposition 3.5, we get

$$
\begin{aligned}
\mathbb{E}\left[\left\|u^{n+1}\right\|^{2}\right] & \leq \frac{1}{1+k^{2} \lambda_{1}^{2}}\left(\mathbb{E}\left[\left\|u^{n}\right\|^{2}\right]+k \operatorname{Tr}(Q)\right) \\
& \leq \mathbb{E}\left[\left\|u^{0}\right\|^{2}\right]+\frac{\operatorname{Tr}(Q)}{k \lambda_{1}^{2}},
\end{aligned}
$$

where $\lambda_{1}$ is the smallest eigenvalue of the Laplacian.

3. Let $\left(\lambda_{j}, e_{j}\right)$ be the eigenpairs of the Laplace operator and let $\left(\alpha_{l}, f_{l}\right)$ be the eigenpairs of the covariance operator $Q$. Writing $u(t)=\sum_{j=1}^{\infty} c_{j}(t) e_{j}$ we have that the expected mass is given by

$$
\mathbb{E}[M(u(t))]=\sum_{j=1}^{\infty} \mathbb{E}\left[\left|c_{j}(t)\right|^{2}\right]
$$

The midpoint rule thus becomes

$$
\sum_{j=1}^{\infty}\left(1+\frac{\mathrm{i} k \lambda_{j}}{2}\right) c_{j}^{n+1} e_{j}=\sum_{j=1}^{\infty}\left(1-\frac{\mathrm{i} k \lambda_{j}}{2}\right) c_{j}^{n} e_{j}-\mathrm{i} \sum_{j=1}^{\infty} \sum_{l=1}^{\infty} \alpha_{l} \Delta \beta_{l}^{n}\left(f_{l}, e_{j}\right) e_{j} .
$$

Thus, for every $j \geq 1$ we have

$$
\left(1+\frac{\mathrm{i} k \lambda_{j}}{2}\right) c_{j}^{n+1}=\left(1-\frac{\mathrm{i} k \lambda_{j}}{2}\right) c_{j}^{n}-\mathrm{i} \sum_{l=1}^{\infty} \alpha_{l} \Delta \beta_{l}^{n}\left(f_{l}, e_{j}\right) .
$$

Multiplying both sides with the conjugate and taking the expectation, we have

$$
\begin{aligned}
\left(1+\frac{k^{2} \lambda_{j}^{2}}{4}\right) \mathbb{E}\left[\left|c_{j}^{n+1}\right|^{2}\right] & =\left(1+\frac{k^{2} \lambda_{j}^{2}}{4}\right) \mathbb{E}\left[\left|c_{j}^{n}\right|^{2}\right]+\sum_{l=1}^{\infty}\left|\alpha_{l}\right|^{2} \mathbb{E}\left[\left|\Delta \beta_{l}^{n}\right|^{2}\right]\left|\left(f_{l}, e_{j}\right)\right|^{2} \\
& =\left(1+\frac{k^{2} \lambda_{j}^{2}}{4}\right) \mathbb{E}\left[\left|c_{j}^{n}\right|^{2}\right]+k \sum_{l=1}^{\infty}\left|\alpha_{l}\right|^{2}\left|\left(f_{l}, e_{j}\right)\right|^{2} .
\end{aligned}
$$


Using that $\left\{\lambda_{j}\right\}_{j=1}^{\infty}$ is a positive and increasing sequence, we get

$$
\begin{aligned}
\mathbb{E}\left[\left|c_{j}^{n+1}\right|^{2}\right] & =\mathbb{E}\left[\left|c_{j}^{n}\right|^{2}\right]+\frac{k}{1+\frac{k^{2} \lambda_{j}}{4}} \sum_{l=1}^{\infty}\left|\alpha_{l}\right|^{2}\left|\left(f_{l}, e_{j}\right)\right|^{2} \\
& =\mathbb{E}\left[\left|c_{j}^{0}\right|^{2}\right]+\frac{t_{n+1}}{1+\frac{k^{2} \lambda_{j}}{4}} \sum_{l=1}^{\infty}\left|\alpha_{l}\right|^{2}\left|\left(f_{l}, e_{j}\right)\right|^{2} \\
& \leq \mathbb{E}\left[\left|c_{j}^{0}\right|^{2}\right]+\frac{t_{n+1}}{1+\frac{k^{2} \lambda_{1}}{4}} \sum_{l=1}^{\infty}\left|\alpha_{l}\right|^{2}\left|\left(f_{l}, e_{j}\right)\right|^{2} .
\end{aligned}
$$

Now using Parseval's identity, we finally obtain

$$
\begin{aligned}
\mathbb{E}\left[M\left(u^{n+1}\right)\right]=\sum_{j=0}^{\infty} \mathbb{E}\left[\left|c_{j}^{n+1}\right|^{2}\right] & \leq \sum_{j=0}^{\infty} \mathbb{E}\left[\left|c_{j}^{0}\right|^{2}\right]+\frac{t_{n+1}}{1+\frac{k^{2} \lambda_{1}}{4}} \sum_{l=1}^{\infty}\left|\alpha_{l}\right|^{2} \sum_{j=0}^{\infty}\left|\left(f_{l}, e_{j}\right)\right|^{2} \\
& =\sum_{j=0}^{\infty} \mathbb{E}\left[\left|c_{j}^{0}\right|^{2}\right]+\frac{t_{n+1}}{1+\frac{k^{2} \lambda_{1}}{4}} \sum_{l=1}^{\infty}\left|\alpha_{l}\right|^{2}\left\|f_{i}\right\|^{2} \\
& =\sum_{j=0}^{\infty} \mathbb{E}\left[\left|c_{j}^{0}\right|^{2}\right]+\frac{t_{n+1}}{1+\frac{k^{2} \lambda_{1}}{4}} \sum_{l=1}^{\infty}\left|\alpha_{l}\right|^{2} \\
& =\mathbb{E}\left[M\left(u^{0}\right)\right]+\frac{t_{n+1}}{1+\frac{k^{2} \lambda_{1}}{4}} \operatorname{Tr}(Q) .
\end{aligned}
$$

$\square$

3.3. A trace formula for the energy. Again, under appropriate boundary conditions, for example periodic boundary conditions or homogeneous Dirichlet boundary conditions, it is well known that the energy

$$
H(u(t)):=\frac{1}{2} \int|\nabla u|^{2} \mathrm{~d} x
$$

of the deterministic linear Schrödinger equation $\mathrm{i} \frac{\partial u}{\partial t}-\Delta u=0$ remains constant along the exact solution, see for example [6]. When additive noise is introduced into the problem, we get a linear drift in the expected value of the total energy as stated in the following result.

Proposition 3.7. Assume that the initial data is such that $\mathbb{E}\left[H\left(u_{0}\right)\right]$ is finite, and that $\left\|\nabla Q^{1 / 2}\right\|_{\mathscr{L}_{2}^{0}}$ is bounded. Then the exact solution of the linear Schrödinger equation with additive noise (3.1) satisfies the trace formula for the expected energy

$$
\mathbb{E}[H(u(t))]=\mathbb{E}\left[H\left(u_{0}\right)\right]+\frac{t}{2} \operatorname{Tr}(\nabla Q \nabla) \quad \text { for all time } \quad t .
$$


Proof. Using Lemma 2.1 and the fact that the Ito integral is normally distributed with mean 0 , we have

$$
\begin{aligned}
\mathbb{E}\left[\|\nabla u(t)\|_{L^{2}}^{2}\right]= & \mathbb{E}\left[\left\|\nabla\left(S(t) u_{0}\right)-\mathrm{i} \int_{0}^{t} \nabla S(t-r) \mathrm{d} W(r)\right\|_{L^{2}}^{2}\right] \\
= & \mathbb{E}\left[\left\|\nabla\left(S(t) u_{0}\right)\right\|_{L^{2}}^{2}+\left\|\int_{0}^{t} \nabla S(t-r) \mathrm{d} W(r)\right\|_{L^{2}}^{2}\right. \\
& \left.-\left(\nabla\left(S(t) u_{0}\right), \mathrm{i} \int_{0}^{t} \nabla S(t-r) \mathrm{d} W(r)\right)-\left(\mathrm{i} \int_{0}^{t} \nabla S(t-r) \mathrm{d} W(r), \nabla\left(S(t) u_{0}\right)\right)\right] \\
= & \mathbb{E}\left[\left\|\nabla u_{0}\right\|_{L^{2}}^{2}\right]+\int_{0}^{t} \mathbb{E}\left[\left\|\nabla\left(S(t-r) Q^{1 / 2}\right)\right\|_{\mathscr{L}_{2}^{0}}^{2}\right] \mathrm{d} r \\
= & \mathbb{E}\left[\left\|\nabla u_{0}\right\|_{L^{2}}^{2}\right]+t \operatorname{Tr}(\nabla Q \nabla) .
\end{aligned}
$$

口

We now show that our exponential integrator satisfies the very same energy trace formula as the exact solution to the linear stochastic Schrödinger equation (3.1).

Proposition 3.8. With the same assumptions as in Proposition 3.7 the exponential integrator (3.2) satisfies the following trace formula

$$
\begin{aligned}
\mathbb{E}\left[H\left(u^{n}\right)\right] & =\mathbb{E}\left[H\left(u^{n-1}\right)\right]+\frac{k}{2} \operatorname{Tr}(\nabla Q \nabla) \\
& =\mathbb{E}\left[H\left(u_{0}\right)\right]+\frac{t_{n}}{2} \operatorname{Tr}(\nabla Q \nabla) \text { for all } t_{n}=n k
\end{aligned}
$$

Proof. Similarly to the proof of the previous proposition, we have

$$
\begin{aligned}
\mathbb{E}\left[H\left(u^{n}\right)\right] & =\frac{1}{2} \mathbb{E}\left[\left\|\nabla u^{n}\right\|_{L^{2}}^{2}\right] \\
& =\frac{1}{2} \mathbb{E}\left[\left\|\nabla\left(S(k) u^{n-1}\right)-\mathrm{i} \int_{t_{n-1}}^{t_{n}} \nabla S(k) \mathrm{d} W(r)\right\|_{L^{2}}^{2}\right] \\
& =\frac{1}{2} \mathbb{E}\left[\left\|\nabla u^{n-1}\right\|_{L^{2}}^{2}\right]+\frac{1}{2} \int_{t_{n-1}}^{t_{n}} \mathbb{E}\left[\left\|\nabla\left(S(k) Q^{1 / 2}\right)\right\|_{\mathscr{L}_{2}^{0}}^{2}\right] \mathrm{d} r \\
& =\mathbb{E}\left[H\left(u^{n-1}\right)\right]+\frac{k}{2} \operatorname{Tr}(\nabla Q \nabla) .
\end{aligned}
$$

3.4. A formula for the momentum. In the deterministic case, one has an additional conserved quantity, the momentum

$$
p(u):=\mathrm{i} \int(u \nabla \bar{u}-\bar{u} \nabla u) \mathrm{d} x .
$$

The next result investigate the behaviour of this quantity in the stochastic case.

Proposition 3.9. Assume that $\mathbb{E}\left[p\left(u_{0}\right)\right]<\infty$ and $Q^{1 / 2} \in \mathscr{L}_{2}^{1}$. Then the exact solution of the linear Schrödinger equation with additive noise (3.1) exhibits the following formula for the expected momentum

$$
\mathbb{E}[p(u(t))]=\mathbb{E}\left[p\left(u_{0}\right)\right]-2 t \operatorname{Im}\left\langle Q^{1 / 2}, \nabla Q^{1 / 2}\right\rangle_{\mathscr{L}_{2}^{0}} \quad \text { for all time } \quad t
$$


Here, we denote by $\langle\cdot, \cdot\rangle$ the usual $L^{2}$ inner product

$$
\langle u, v\rangle:=\int_{\mathbb{R}^{d}} u \bar{v} \mathrm{~d} x,
$$

for $u, v \in L^{2}$. And further denote the Hilbert-Schmidt inner product from $L^{2}$ to $L^{2}$ with the above inner product by $\langle\cdot, \cdot\rangle_{\mathscr{L}_{2}^{0}}$.

Proof. The momentum can be written as

$$
p(u(t))=\mathrm{i}\langle u(t), \nabla u(t)\rangle-\mathrm{i}\langle\nabla u(t), u(t)\rangle .
$$

Using the mild solution, we have

$$
\begin{aligned}
\langle u(t), \nabla u(t)\rangle= & \left\langle S(t) u_{0}-\mathrm{i} \int_{0}^{t} S(t-r) \mathrm{d} W(r), \nabla S(t) u_{0}-\mathrm{i} \int_{0}^{t} \nabla S(t-r) \mathrm{d} W(r)\right\rangle \\
= & \left\langle S(t) u_{0}, \nabla S(t) u_{0}\right\rangle+\left\langle S(t) u_{0},-\mathrm{i} \int_{0}^{t} S(t-r) \nabla \mathrm{d} W(r)\right\rangle \\
& +\left\langle-\mathrm{i} \int_{0}^{t} S(t-r) \mathrm{d} W(r), S(t) \nabla u_{0}\right\rangle \\
& +\left\langle-\mathrm{i} \int_{0}^{t} S(t-r) \mathrm{d} W(r),-\mathrm{i} \int_{0}^{t} S(t-r) \nabla \mathrm{d} W(r)\right\rangle .
\end{aligned}
$$

Taking the expectation, the terms containing one integral is zero. Using also the Ito isometry we obtain

$$
\begin{aligned}
\mathbb{E}[\langle u(t), \nabla u(t)\rangle] & =\mathbb{E}\left[\left\langle S(t) u_{0}, \nabla S(t) u_{0}\right\rangle\right]+\mathbb{E}\left[\left\langle\int_{0}^{t} S(t-r) \mathrm{d} W(r), \int_{0}^{t} S(t-r) \nabla \mathrm{d} W(r)\right\rangle\right] \\
& =\mathbb{E}\left[\left\langle u_{0}, \nabla u_{0}\right\rangle\right]+\mathbb{E}\left[\int_{0}^{t}\left\langle S(t-r) Q^{1 / 2}, S(t-r) \nabla Q^{1 / 2}\right\rangle_{\mathscr{L}_{2}^{0}} \mathrm{~d} r\right] \\
& =\mathbb{E}\left[\left\langle u_{0}, \nabla u_{0}\right\rangle\right]+\mathbb{E}\left[\int_{0}^{t}\left\langle Q^{1 / 2}, \nabla Q^{1 / 2}\right\rangle_{\mathscr{L}_{2}^{0}} \mathrm{~d} r\right] \\
& =\mathbb{E}\left[\left\langle u_{0}, \nabla u_{0}\right\rangle\right]+t\left\langle Q^{1 / 2}, \nabla Q^{1 / 2}\right\rangle_{\mathscr{L}_{2}^{0}} .
\end{aligned}
$$

Similarly, we have

$$
\mathbb{E}[\langle\nabla u(t), u(t)\rangle]=\mathbb{E}\left[\left\langle\nabla u_{0}, u_{0}\right\rangle\right]+t\left\langle\nabla Q^{1 / 2}, Q^{1 / 2}\right\rangle_{\mathscr{L}_{2}^{0}} .
$$

For the expected momentum we finally obtain

$$
\begin{aligned}
\mathbb{E}[p(u(t))] & =\mathbb{E}[\mathrm{i}\langle u(t), \nabla u(t)\rangle-\mathrm{i}\langle\nabla u(t), u(t)\rangle] \\
& =\mathrm{i} \mathbb{E}\left[\left\langle u_{0}, \nabla u_{0}\right\rangle-\left\langle\nabla u_{0}, u_{0}\right\rangle\right]+\mathrm{i} t\left(\left\langle Q^{1 / 2}, \nabla Q^{1 / 2}\right\rangle_{\mathscr{L}_{2}^{0}}-\left\langle\nabla Q^{1 / 2}, Q^{1 / 2}\right\rangle_{\mathscr{L}_{2}^{0}}\right) \\
& =\mathbb{E}\left[p\left(u_{0}\right)\right]+\mathrm{i} t\left(\left\langle Q^{1 / 2}, \nabla Q^{1 / 2}\right\rangle_{\mathscr{L}_{2}^{0}}-\overline{\left\langle Q^{1 / 2}, \nabla Q^{1 / 2}\right\rangle_{\mathscr{L}_{2}^{0}}}\right) \\
& =\mathbb{E}\left[p\left(u_{0}\right)\right]-2 t \operatorname{Im}\left\langle Q^{1 / 2}, \nabla Q^{1 / 2}\right\rangle_{\mathscr{L}_{2}^{0}} .
\end{aligned}
$$

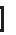


As we see in the proposition below, the exponential method satisfies the same trace formula for the momentum.

Proposition 3.10. With the same assumptions as in Proposition 3.9 the exponential integrator 3.2 exhibits the following formula for the expected momentum.

$$
\mathbb{E}\left[p\left(u^{n}\right)\right]=\mathbb{E}\left[p\left(u_{0}\right)\right]-2 t_{n} \operatorname{Im}\left\langle Q^{1 / 2}, \nabla Q^{1 / 2}\right\rangle_{\mathscr{L}_{2}^{0}} \text { for all } t_{n}=n k
$$

Proof. Writing

$$
u^{n}=S(k) u^{n-1}-\mathrm{i} \int_{t_{n-1}}^{t_{n}} S(k) \mathrm{d} W(r)
$$

we can use the same techniques as in the proof for the exact solution. We obtain

$$
\begin{aligned}
\mathbb{E}\left[p\left(u^{n}\right)\right] & =\mathbb{E}\left[p\left(u^{n-1}\right)\right]-2 k \operatorname{Im}\left\langle Q^{1 / 2}, \nabla Q^{1 / 2}\right\rangle_{\mathscr{L}_{2}^{0}} \\
& =\mathbb{E}\left[p\left(u_{0}\right)\right]-2 t_{n} \operatorname{Im}\left\langle Q^{1 / 2}, \nabla Q^{1 / 2}\right\rangle_{\mathscr{L}_{2}^{0}}
\end{aligned}
$$

3.5. Numerical experiments for the linear stochastic Schrödinger equation. This subsection illustrates the above properties (error estimates and trace formulas) of the stochastic exponential integrator (3.2) when applied to the linear stochastic Schrödinger equation 3.1.

Let us first consider the error in the time integration of the linear stochastic Schrödinger equation. For this, we consider problem (3.1) on the interval $[0,2 \pi]$ with periodic boundary conditions. The initial value is taken to be $u_{0}=0$ and the eigenvalues of the covariance operator $Q$ are given by $\lambda_{n}=1 /\left(1+n^{8}\right)$ for $n \in \mathbb{Z}$. Such a regular noise is needed for the midpoint rule to be convergent, see [17, Proposition 3.1] and the discussion below. The spatial discretisation is done by a pseudospectral method (with $M=2^{8}$ Fourier modes) using the following representation of the noise

$$
W(x, t)=\sum_{n \in \mathbb{Z}} \lambda_{n}^{1 / 2} \beta_{n}(t) e_{n}(x)
$$

where $\left\{\beta_{n}(t)\right\}_{n \in \mathbb{Z}}$ are i.i.d Brownian motion and $\left\{e_{n}(x)\right\}_{n \in \mathbb{Z}}=\left\{\frac{1}{\sqrt{2 \pi}} \mathrm{e}^{\mathrm{i} n x}\right\}_{n \in \mathbb{Z}}$ is an orthonormal basis of $L^{2}(0,2 \pi)$. The resulting system of stochastic differential equations is then integrated in time by the stochastic exponential integrator 3.2) (SEXP), the Crank-Nicolson scheme from [17, Section 3], which reduces to the stochastic implicit midpoint rule (MP), and the classical backward Euler-Maruyama methods (BEM). Note that the classical explicit Euler-Maruyama would require an unreasonable small time step and is therefore omitted in our computational experiments. Observe also that MP and BEM are semi-implicit methods whereas SEXP is explicit. The rates of mean-square convergence (measured in the $L^{2}$-norm at the end of the interval of integration $[0,0.5]$ ) of these integrators are presented in Figure 3.1. The expected rate of convergence $\mathscr{O}\left(k^{2}\right)$ of the stochastic exponential integrator, as stated in Theorem 3.1, can be confirmed. Here, the exact solution is approximated by the stochastic midpoint rule with a very small time step $k_{\text {exact }}=2^{-10}$ and $M_{\mathrm{s}}=750000$ samples are used for the approximation of the expected values. Note that here and in all our numerical experiments, enough samples are taken in order for the Monte-Carlo errors to be negligible. 


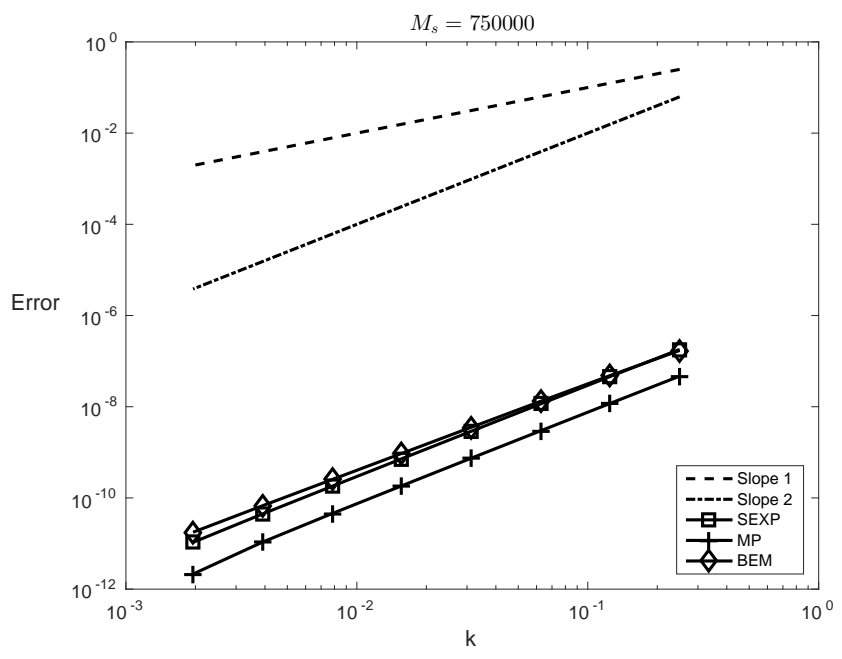

FIGURE 3.1. Linear stochastic Schrödinger equation: Mean-square errors for the stochastic exponential integrator (SEXP), the midpoint rule (MP), and backward Euler-Maruyama (BEM). The dotted, resp. dash-dotted, lines have slopes 1 and 2 .

We now turn to the trace formulas. Figure 3.2 illustrates the energy trace formula from Proposition 3.7 and compare the results obtained with our stochastic exponential method, with the backward Euler-Maruyama method, and with the midpoint rule. $M=2^{7}$ Fourier modes are used for the spatial discretisation; $M_{\mathrm{S}}=10000$ samples are used for the approximatation of the expected values; the time interval is [0,2500], and all schemes use a step size $k=0.1$. The other parameters are the same as in the above numerical experiments. The numerical linear drift in the expected value of the energy of the stochastic exponential integrator (3.2), as stated in Proposition 3.8, is observed in this figure. One also observes an excellent behaviour of the midpoint rule in reproducing the linear growth of the expected energy correctly. This is in contrast with the wrong behaviour of the backward Euler-Maruyama method. Note that this fact was already observed for the trace formula for the linear stochastic wave equation [9].

Using the same parameters as in the previous numerical experiments, a similar behaviour of the numerical methods is observed for the mass trace formula given in Proposition 3.2 These results are not displayed. However, when considering a less regular noise, for example when the eigenvalues of $Q$ are given by $\lambda_{n}=1 /\left(1+n^{2}\right)$, which is of trace-class, one observes that the midpoint rule does not perform as well as the stochastic exponential method, see Figure 3.3. This further illustrates the theoretical results obtained in Proposition 3.5.

4. Linear stochastic Schrödinger equations with a multiplicative potential. We now consider the stochastic Schrödinger equation

$$
\begin{array}{ll}
\mathrm{id} u=\Delta u \mathrm{~d} t+V(x) u \mathrm{~d} t+\mathrm{d} W & \text { in } \mathbb{R}^{d} \times(0, \infty), \\
u(\cdot, 0)=u_{0}, & \text { in } \mathbb{R}^{d},
\end{array}
$$

where $V(x)$ is a real-valued potential. The initial data $u_{0}$ is an $\mathscr{F}_{0}$-measurable complexvalued random variable, and the $Q$-Wiener process $\{W(t)\}_{t \geq 0}$ is again complex-valued and square integrable with respect to the filtration. Further assumptions on $d, u_{0}, V(x)$ and $Q$ will be made when the results of this section are presented. In this section we aim to prove convergence rate of our exponential integrator, trace formulas for the mass and energy, and 


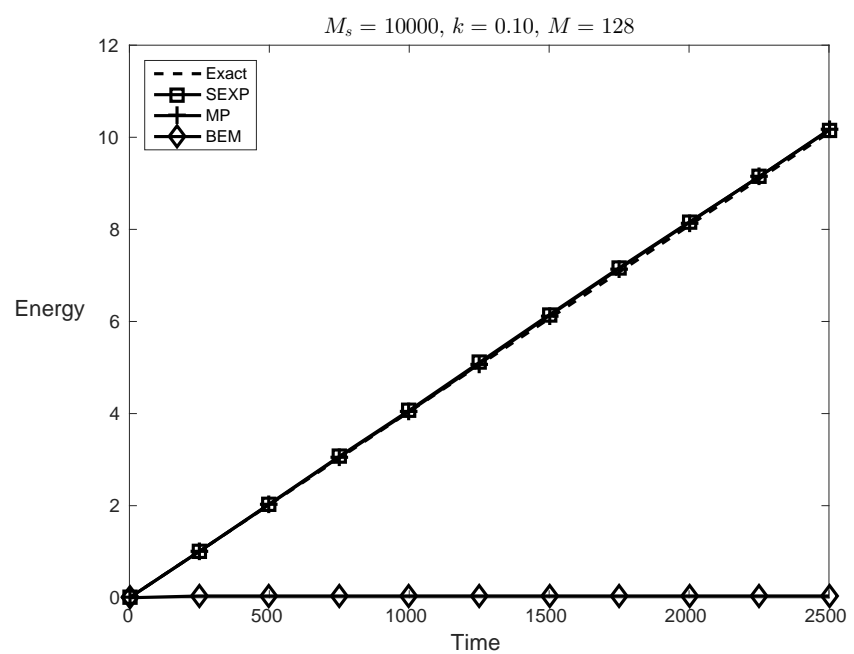

FIGURE 3.2. Linear stochastic Schrödinger equation: Energy trace formulas for the numerical solutions given by the stochastic exponential method (SEXP), the midpoint rule (MP) and the backward Euler-Maruyama scheme $(B E M)$. Time interval and time step: $[0,2500]$, resp. $k=0.1$.

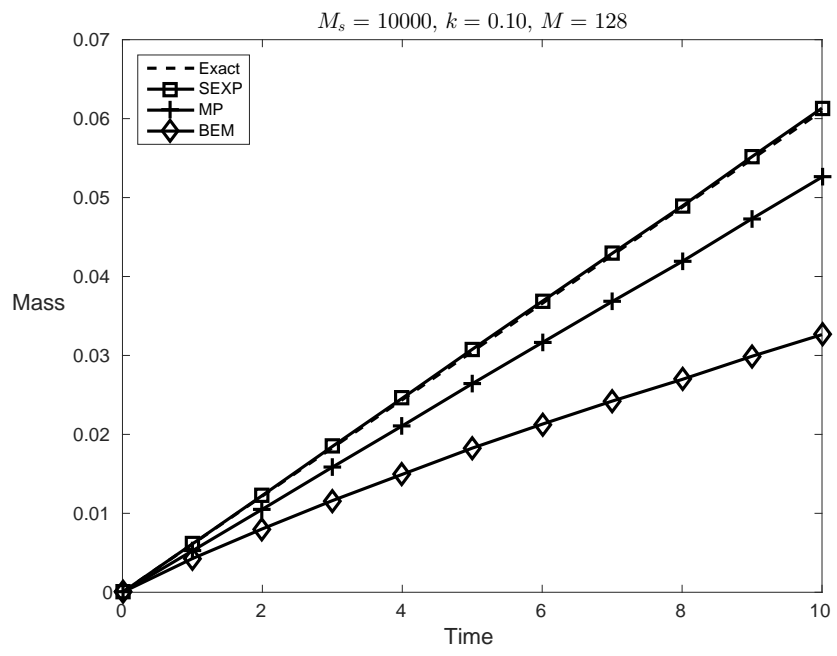

FIGURE 3.3. Linear stochastic Schrödinger equation: Mass trace formulas for the numerical solutions given by the stochastic exponential method (SEXP), the midpoint rule (MP) and the backward Euler-Maruyama scheme (BEM). Time interval and time step: $[0,10]$, resp. $k=0.1$.

finally present numerical experiments that confirm these theoretical findings.

We use the same semigroup approach as in Section 3 and write the mild equation of 4.1 as

$$
u(t)=S(t) u_{0}-\mathrm{i} \int_{0}^{t} S(t-r) V(x) u(r) \mathrm{d} r-\mathrm{i} \int_{0}^{t} S(t-r) \mathrm{d} W(r),
$$

where we recall that $S(t)=\mathrm{e}^{-\mathrm{i} t \Delta}$. We have the following result regarding existence, uniqueness and boundedness of solutions of $4.1 \mathrm{p}$.

Proposition 4.1. Assume that $\mathbb{E}\left[\left\|u_{0}\right\|_{\sigma}^{2 p}\right]<\infty$ for some $p \in \mathbb{N}$, and $Q^{1 / 2} \in \mathscr{L}_{2}^{\sigma}$. Assume also that 
(i) $\sigma>\frac{d}{2}$ and $V(x) \in H^{\sigma}$, or

(ii) $\sigma=0$ and $d=1,2,3$ and $V(x) \in H^{2}$, or

(iii) $\sigma=1$ and $d=2,3$ and $V(x) \in H^{3}$.

Then (4.1) has a unique solution on $[0, T]$, for $T \in(0, \infty)$, which is given by 4.2] and satisfies

$$
\mathbb{E}\left[\sup _{0 \leq t \leq T}\|u(t)\|_{\sigma}^{2 p}\right] \leq C
$$

Proof. We first observe that with the different assumptions (i)-(iii), we have

(i) $H^{\sigma}$ forms an algebra. In particular we have, for example, $\|V(x) u(t)\|_{\sigma} \leq C\|V(x)\|_{\sigma}\|u(t)\|_{\sigma}$.

(ii) In this case we have $H^{2} \subset L^{\infty}$, so whenever $V(x) \in H^{2}$ we have $\|V(x) u(t)\|_{L^{2}} \leq$ $\|V(x)\|_{L^{\infty}}\|u(t)\|_{L^{2}} \leq C\|u(t)\|_{L^{2}}$.

(iii) We still have $H^{2} \subset L^{\infty}$ and, if $V(x) \in H^{3}$, then $\|V(x) u(r)\|_{1} \leq C\|u(r)\|_{1}$. This can be seen by the following calculation:

$$
\begin{aligned}
\|V(x) u(r)\|_{1}^{2} & =\|V(x) u(r)\|_{L^{2}}^{2}+\|\nabla(V(x) u(r))\|_{L^{2}}^{2} \\
& =\|V(x) u(r)\|_{L^{2}}^{2}+\|\nabla V(x) \cdot u(r)+V(x) \nabla u(r)\|_{L^{2}}^{2} \\
& \leq\|V(x) u(r)\|_{L^{2}}^{2}+2\left(\|\nabla V(x) \cdot u(r)\|_{L^{2}}^{2}+\|V(x) \nabla u(r)\|_{L^{2}}^{2}\right) \\
& \leq 3\|V(x)\|_{L^{\infty}}^{2}\left(\|u(r)\|_{L^{2}}^{2}+\|\nabla u(r)\|_{L^{2}}^{2}\right)+2\|\nabla V(x)\|_{L^{\infty}}^{2}\|u(r)\|_{L^{2}}^{2} \\
& \leq C\|u(r)\|_{1}^{2},
\end{aligned}
$$

since $\|\nabla V(x)\|_{L^{\infty}}<\infty$ if $V(x) \in H^{3}$.

Existence and uniqueness now follow from a standard fixed point argument (see [11, Theorem 7.4]) using what was mentioned above about the different cases (i)-(iii). To prove boundedness, we use the mild equation (4.2).

$$
\begin{aligned}
\mathbb{E}\left[\sup _{0 \leq t \leq T}\|u(t)\|_{\sigma}^{2 p}\right] \leq & C\left(\mathbb{E}\left[\sup _{0 \leq t \leq T}\left\|S(t) u_{0}\right\|_{\sigma}^{2 p}\right]+\mathbb{E}\left[\sup _{0 \leq t \leq T}\left\|\int_{0}^{t} S(t-r) V(x) u(r) \mathrm{d} r\right\|_{\sigma}^{2 p}\right]\right. \\
& \left.+\mathbb{E}\left[\sup _{0 \leq t \leq T}\left\|\int_{0}^{t} S(t-r) \mathrm{d} W(r)\right\|_{\sigma}^{2 p}\right]\right) .
\end{aligned}
$$

We now proceed by estimating the terms on the right-hand side. First we have, by Lemma 2.1 .

$$
\mathbb{E}\left[\sup _{0 \leq t \leq T}\left\|S(t) u_{0}\right\|_{\sigma}^{2 p}\right]=\mathbb{E}\left[\left\|u_{0}\right\|_{\sigma}^{2 p}\right] \leq C
$$

For the second term we need to treat each case (i)-(iii) separately. 
(i) Using Lemma 2.1 the fact that $H^{\sigma}$ is an algebra, and Hölder's inequality, we have

$$
\begin{aligned}
\mathbb{E}\left[\sup _{0 \leq t \leq T}\left\|\int_{0}^{t} S(t-r) V(x) u(r) \mathrm{d} r\right\|_{\sigma}^{2 p}\right] & \leq \mathbb{E}\left[\sup _{0 \leq t \leq T}\left(\int_{0}^{t}\|S(t-r) V(x) u(r)\|_{\sigma} \mathrm{d} r\right)^{2 p}\right] \\
& \leq \mathbb{E}\left[\sup _{0 \leq t \leq T}\left(\int_{0}^{t}\|V(x)\|_{\sigma}\|u(r)\|_{\sigma} \mathrm{d} r\right)^{2 p}\right] \\
& \leq C\|V(x)\|_{\sigma}^{2 p} \mathbb{E}\left[\sup _{0 \leq t \leq T}\left(\int_{0}^{t}\|u(r)\|_{\sigma} \mathrm{d} r\right)^{2 p}\right] \\
& \leq C \mathbb{E}\left[\sup _{0 \leq t \leq T}\left(\left(\int_{0}^{t}\|u(r)\|_{\sigma}^{2 p} \mathrm{~d} r\right)^{\frac{1}{2 p}}\left(\int_{0}^{t} 1^{\frac{2 p}{2 p-1}} \mathrm{~d} r\right)^{\frac{2 p-1}{2 p}}\right)^{2 p}\right] \\
& \leq C T^{2 p-1} \mathbb{E}\left[\sup _{0 \leq t \leq T} \int_{0}^{t}\|u(r)\|_{\sigma}^{2 p} \mathrm{~d} r\right] \\
& \leq C \int_{0}^{T} \mathbb{E}\left[\sup _{0 \leq s \leq r}\|u(s)\|_{\sigma}^{2 p}\right] \mathrm{d} r .
\end{aligned}
$$

In the last step we have used that $\|u(r)\|_{\sigma}^{2 p} \leq \sup _{0 \leq s \leq r}\|u(s)\|_{\sigma}^{2 p}$.

(ii) If instead $\sigma=0$ and $d=1,2,3$ and thus $H^{\sigma}=L^{2}$ is not an algebra, then we have, using $H^{2} \subset L^{\infty}$,

$$
\begin{aligned}
\mathbb{E}\left[\sup _{0 \leq t \leq T}\left(\int_{0}^{t}\|S(t-r) V(x) u(r)\|_{L^{2}} \mathrm{~d} r\right)^{2 p}\right] & \leq\|V(x)\|_{L^{\infty}}^{2 p} \mathbb{E}\left[\sup _{0 \leq t \leq T}\left(\int_{0}^{t}\|u(r)\|_{L^{2}} \mathrm{~d} r\right)^{2 p}\right] \\
& \leq C \mathbb{E}\left[\sup _{0 \leq t \leq T}\left(\int_{0}^{t}\|u(r)\|_{L^{2}} \mathrm{~d} r\right)^{2 p}\right]
\end{aligned}
$$

The same estimate follows from similar calculations as in (i).

(iii) We now assume $\sigma=1, d=2,3$ and $V(x) \in H^{3}$. The proof follows the same lines as in (ii), noting that

$$
\|V(x) u(r)\|_{1} \leq C\|u(r)\|_{1},
$$

as seen above.

For the third and last term, using Burkholder's inequality, we obtain

$$
\begin{aligned}
\mathbb{E}\left[\sup _{0 \leq t \leq T}\left\|\int_{0}^{t} S(t-r) \mathrm{d} W(r)\right\|_{\sigma}^{2 p}\right] & \leq C \mathbb{E}\left[\left(\int_{0}^{T}\left\|Q^{1 / 2}\right\|_{\mathscr{L}_{2}^{\sigma}}^{2} \mathrm{~d} t\right)^{p}\right] \\
& \leq C T^{p} \mathbb{E}\left[\left\|Q^{1 / 2}\right\|_{\mathscr{L}_{2}^{\sigma}}^{2 p}\right] \leq C .
\end{aligned}
$$

Altogether we arrive at

$$
\mathbb{E}\left[\sup _{0 \leq t \leq T}\|u(t)\|_{\sigma}^{2 p}\right] \leq C_{1}+C_{2} \int_{0}^{T} \mathbb{E}\left[\sup _{0 \leq s \leq t}\|u(s)\|_{\sigma}^{2 p}\right] \mathrm{d} t,
$$

and an application of Gronwall's lemma completes the proof. $\mathrm{Y}$ 
4.1. Error analysis of the stochastic exponential method. Our exponential scheme for the time integration of 4.1) now reads

$$
\begin{aligned}
u^{n} & =S(k) u^{n-1}-\mathrm{i} k S(k) V(x) u^{n-1}-\mathrm{i} S(k) \Delta W^{n-1} \\
& =S\left(t_{n}\right) u_{0}-\mathrm{i} \sum_{j=0}^{n-1} \int_{t_{j}}^{t_{j+1}} S\left(t_{n}-t_{j}\right) V(x) u^{j} \mathrm{~d} r-\mathrm{i} \sum_{j=0}^{n-1} \int_{t_{j}}^{t_{j+1}} S\left(t_{n}-t_{j}\right) \mathrm{d} W(r),
\end{aligned}
$$

where, as before, $k$ denotes the step size, $t_{n}=n k$ for $n=0, \ldots, N$, and $\Delta W^{n-1}=W\left(t_{n}\right)-$ $W\left(t_{n-1}\right)$. We first show that these numerical approximations are bounded.

Proposition 4.2. Assume that $\mathbb{E}\left[\left\|u_{0}\right\|_{\sigma}^{2 p}\right]<\infty$ for some $p \in \mathbb{N}$, and $Q^{1 / 2} \in \mathscr{L}_{2}^{\sigma}$. As in Proposition 4.1 we also assume

(i) $\sigma>\frac{d}{2}$, and $V(x) \in H^{\sigma}$, or

(ii) $\sigma=0$ and $d=1,2,3$, and $V(x) \in H^{2}$, or

(iii) $\sigma=1$ and $d=2,3$, and $V(x) \in H^{3}$.

Then the numerial solution given by (4.3), with step size $k$, satisfies

$$
\mathbb{E}\left[\left\|u^{n}\right\|_{\sigma}^{2 p}\right] \leq C \quad \text { for } \quad 0 \leq t_{n}=n k \leq T .
$$

Proof. We prove the case (i), the other cases are treated as in the proof of Proposition 4.1 We have

$$
\begin{aligned}
\mathbb{E}\left[\left\|u^{n}\right\|_{\sigma}^{2 p}\right] \leq & C\left(\mathbb{E}\left[\left\|S\left(t_{n}\right) u_{0}\right\|_{\sigma}^{2 p}\right]+\mathbb{E}\left[\left\|\mathrm{i} k \sum_{j=0}^{n-1} S\left(t_{n}-t_{j}\right) V(x) u^{j}\right\|_{\sigma}^{2 p}\right]\right. \\
& \left.+\mathbb{E}\left[\left\|\mathrm{i} \sum_{j=0}^{n-1} \int_{t_{j}}^{t_{j+1}} S\left(t_{n}-t_{j}\right) \mathrm{d} W(r)\right\|_{\sigma}^{2 p}\right]\right) .
\end{aligned}
$$

The first term is estimated as in Proposition 4.1 For the second term, using Hölder's inequality and the fact that $V(x) \in H^{\sigma}$, we have

$$
\begin{aligned}
\left\|k \sum_{j=0}^{n-1} S\left(t_{n}-t_{j}\right) V(x) u^{j}\right\|_{\sigma} & \leq k \sum_{j=0}^{n-1}\left\|V(x) u^{j}\right\|_{\sigma} \\
& \leq C k \sum_{j=0}^{n-1}\left\|u^{j}\right\|_{\sigma} \\
& \leq C k^{\frac{1}{2 p}}\left(\sum_{j=0}^{n-1}\left\|u^{j}\right\|_{\sigma}^{2 p}\right)^{\frac{1}{2 p}} .
\end{aligned}
$$

For the third term we use the Burkholder inequality and obtain

$$
\begin{aligned}
\mathbb{E}\left[\left\|\sum_{j=0}^{n-1} \int_{t_{j}}^{t_{j+1}} S\left(t_{n}-t_{j}\right) \mathrm{d} W(r)\right\|_{\sigma}^{2 p}\right] & =\mathbb{E}\left[\left\|\int_{0}^{t_{n}} S\left(t_{n}-[r / k] k\right) \mathrm{d} W(r)\right\|_{\sigma}^{2 p}\right] \\
& \leq C \mathbb{E}\left[\left(\int_{0}^{T}\left\|Q^{1 / 2}\right\|_{\mathscr{L}_{2}^{\sigma}}^{2} \mathrm{~d} t\right)^{p}\right] \\
& \leq C,
\end{aligned}
$$

where, as before, $[r / k]$ denotes the integer part of $r / k$. 
Altogether we arrive at

$$
\mathbb{E}\left[\left\|u^{n}\right\|_{\sigma}^{2 p}\right] \leq C_{1}+C_{2} k \sum_{j=0}^{n-1} \mathbb{E}\left[\left\|u^{j}\right\|_{\sigma}^{2 p}\right]
$$

and an application of Gronwall's lemma concludes the proof.

We are now ready to prove the main result of this section on the convergence of our numerical method.

THEOREM 4.3. Consider the time discretisation of the stochastic Schrödinger equation (4.1) given by the exponential integrator (4.3). Let $d=1,2,3, \sigma \geq 0$, and assume that $\mathbb{E}\left[\left\|u_{0}\right\|_{\sigma+2}^{2 p}\right]<\infty$ for some $p \in \mathbb{N}, V(x) \in H^{\sigma+2}$, and $Q^{1 / 2} \in \mathscr{L}_{2}^{\sigma+2}$. Then there exists a constant $C$ such that

$$
\mathbb{E}\left[\max _{n=1, \ldots, N}\left\|u^{n}-u\left(t_{n}\right)\right\|_{\sigma}^{2 p}\right] \leq C k^{2 p}
$$

REMARK 4.4. Using similar assumptions as in Hypothesis 4.1 and 4.2 in [17], we could replace the term $V(x) u$ in (4.1) by a general Lipschitz function $F(u)$. That is, if we assume $\sigma>\frac{d}{2}$, and $F: H^{\sigma} \rightarrow H^{\sigma}$ is a $C^{2}$ function that is bounded as well as its derivatives up to order 2 , that $\left(F(u(t)) \in L^{2 p}\left(\Omega ; L^{\infty}\left(0, T ; H^{\sigma+2}\right)\right)\right.$, that the solution $(u(t))_{t \in[0, T]}$ is in $L^{2 p}\left(\Omega ; L^{\infty}\left(0, T ; H^{\sigma+2}\right)\right)$ and $L^{4 p}\left(\Omega, L^{\infty}\left(0, T, H^{\sigma+1}\right)\right)$, and $u_{0} \in L^{2 p}\left(\Omega ; H^{\sigma+2}\right)$, then the convergence order stated in Theorem 4.3 remains unchanged.

Proof. As before, there are three cases to consider:

(i) $\sigma>\frac{d}{2}$,

(ii) $\sigma=0$ and $d=1,2,3$,

(iii) $\sigma=1$ and $d=2,3$.

We will prove the case (i) where $\sigma>\frac{d}{2}$, so that $H^{\sigma}$ forms an algebra. In the cases (ii) and (iii), $H^{\sigma}$ does not form an algebra, but these two cases can be treated in a similar way as in the proof of Proposition 4.1 We consider the error

$$
u^{n}-u\left(t_{n}\right)=\operatorname{Err}_{V}^{n}+\operatorname{Err}_{W}^{n}
$$

where we have defined

$$
\operatorname{Err}_{V}^{n}:=\mathrm{i} \sum_{j=0}^{n-1} \int_{t_{j}}^{t_{j+1}}\left(S\left(t_{n}-r\right) V(x) u(r)-S\left(t_{n}-t_{j}\right) V(x) u^{j}\right) \mathrm{d} r
$$

and

$$
\operatorname{Err}_{W}^{n}:=\mathrm{i} \sum_{j=0}^{n-1} \int_{t_{j}}^{t_{j+1}}\left(S\left(t_{n}-r\right)-S\left(t_{n}-t_{j}\right)\right) \mathrm{d} W(r)
$$

This then gives us

$$
\mathbb{E}\left[\max _{n=1, \ldots, N}\left\|u^{n}-u\left(t_{n}\right)\right\|_{\sigma}^{2 p}\right] \leq C\left(\mathbb{E}\left[\max _{n=1, \ldots, N}\left\|\operatorname{Err}_{V}^{n}\right\|_{\sigma}^{2 p}\right]+\mathbb{E}\left[\max _{n=1, \ldots N}\left\|\operatorname{Err}_{W}^{n}\right\|_{\sigma}^{2 p}\right]\right) .
$$

The second term on the right-hand side is the same term as in the proof of Theorem 3.1 and we thus get

$$
\mathbb{E}\left[\max _{n=1, \ldots, N}\left\|\operatorname{Err}_{W}^{n}\right\|_{\sigma}^{2 p}\right] \leq C k^{2 p}
$$


In order to estimate $\operatorname{Err}_{V}^{n}$, we first write it as

$$
\begin{aligned}
\operatorname{Err}_{V}^{n}= & i \sum_{j=0}^{n-1} \int_{t_{j}}^{t_{j+1}} S\left(t_{n}-r\right) V(x)\left(u(r)-u\left(t_{j}\right)\right) \mathrm{d} r \\
& +i \sum_{j=0}^{n-1} \int_{t_{j}}^{t_{j+1}}\left(S\left(t_{n}-r\right)-S\left(t_{n}-t_{j}\right)\right) V(x) u\left(t_{j}\right) \mathrm{d} r \\
& +i \sum_{j=0}^{n-1} \int_{t_{j}}^{t_{j+1}} S\left(t_{n}-t_{j}\right) V(x)\left(u\left(t_{j}\right)-u^{j}\right) \mathrm{d} r \\
= & : I_{1}^{n}+I_{2}^{n}+I_{3}^{n} .
\end{aligned}
$$

The estimate for $I_{1}^{n}$ is more complicated than the estimates for $I_{2}^{n}$ and $I_{3}^{n}$, so we save it for last. Using Lemma2.1 and that $H^{\sigma+2}$ is an algebra, we have

$$
\begin{aligned}
\left\|I_{2}^{n}\right\|_{\sigma} & \leq \sum_{j=0}^{n-1} \int_{t_{j}}^{t_{j+1}}\left\|\left(S\left(t_{n}-r\right)-S\left(t_{n}-t_{j}\right)\right) V(x) u\left(t_{j}\right)\right\|_{\sigma} \mathrm{d} r \\
& =\sum_{j=0}^{n-1} \int_{t_{j}}^{t_{j+1}}\left\|S\left(t_{n}-t_{j}\right)\left(S\left(t_{j}-r\right)-I\right) V(x) u\left(t_{j}\right)\right\|_{\sigma} \mathrm{d} r \\
& \leq \sum_{j=0}^{n-1} \int_{t_{j}}^{t_{j+1}} \mid t_{j}-r\|\| \Delta\left(V(x) u\left(t_{j}\right)\right) \|_{\sigma} \mathrm{d} r \\
& \leq C k^{2} \sum_{j=0}^{n-1}\left\|V(x) u\left(t_{j}\right)\right\|_{\sigma+2} \\
& \leq C k^{2}\|V(x)\|_{\sigma+2} \sum_{j=0}^{n-1}\left\|u\left(t_{j}\right)\right\|_{\sigma+2} .
\end{aligned}
$$

Now, using Hölder's inequality we have

$$
\begin{aligned}
\sum_{j=0}^{n-1}\left\|u\left(t_{j}\right)\right\|_{\sigma+2} & \leq\left(\sum_{j=0}^{n-1}\left\|u\left(t_{j}\right)\right\|_{\sigma+2}^{2 p}\right)^{\frac{1}{2 p}} k^{\frac{1-2 p}{2 p}} \\
& \leq C k^{-1}\left(\sup _{0 \leq t \leq T}\|u(t)\|_{\sigma+2}^{2 p}\right)^{\frac{1}{2 p}} .
\end{aligned}
$$

Thus

$$
\mathbb{E}\left[\max _{n=1, \ldots, N}\left\|I_{2}^{n}\right\|_{\sigma}^{2 p}\right] \leq C k^{2 p} \mathbb{E}\left[\sup _{0 \leq t \leq T}\|u(t)\|_{\sigma+2}^{2 p}\right] \leq C k^{2 p},
$$

by Proposition 4.1 
Again using the fact that $H^{\sigma}$ is an algebra, and then Hölder's inequality, we have

$$
\begin{aligned}
\left\|I_{3}^{n}\right\|_{\sigma} & \leq \sum_{j=0}^{n-1} \int_{t_{j}}^{t_{j+1}}\left\|V(x)\left(u\left(t_{j}\right)-u^{j}\right)\right\|_{\sigma} \mathrm{d} r \\
& \leq C\|V(x)\|_{\sigma} \sum_{j=0}^{n-1} \int_{t_{j}}^{t_{j+1}}\left\|u\left(t_{j}\right)-u^{j}\right\|_{\sigma} \mathrm{d} r \\
& \leq C k \sum_{j=0}^{n-1}\left\|u\left(t_{j}\right)-u^{j}\right\|_{\sigma} \\
& \leq C k\left(\sum_{j=0}^{n-1}\left\|u\left(t_{j}\right)-u^{j}\right\|_{\sigma}^{2 p}\right)^{\frac{1}{2 p}} k^{\frac{1-2 p}{2 p}} \\
& \leq C k^{\frac{1}{2 p}}\left(\sum_{j=0}^{n-1}\left\|u\left(t_{j}\right)-u^{j}\right\|_{\sigma}^{2 p}\right)^{\frac{1}{2 p}}
\end{aligned}
$$

Thus

$$
\mathbb{E}\left[\max _{n=1, \ldots, N}\left\|I_{3}^{n}\right\|_{\sigma}^{2 p}\right] \leq C k \sum_{j=0}^{N-1} \mathbb{E}\left[\max _{l=0, \ldots, j}\left\|u\left(t_{l}\right)-u^{l}\right\|_{\sigma}^{2 p}\right]
$$

In order to estimate $I_{1}^{n}$ we use the mild formulation of the exact solution on the interval $\left[t_{j}, r\right]$

$$
u(r)=S\left(r-t_{j}\right) u\left(t_{j}\right)-\mathrm{i} \int_{t_{j}}^{r} S(r-\rho) V(x) u(\rho) \mathrm{d} \rho-\mathrm{i} \int_{t_{j}}^{r} S(r-\rho) \mathrm{d} W(\rho),
$$

and therefore

$$
u(r)-u\left(t_{j}\right)=\left(S\left(r-t_{j}\right)-I\right) u\left(t_{j}\right)-\mathrm{i} \int_{t_{j}}^{r} S(r-\rho) V(x) u(\rho) \mathrm{d} \rho-\mathrm{i} \int_{t_{j}}^{r} S(r-\rho) \mathrm{d} W(\rho) .
$$

We have

$$
\begin{aligned}
I_{1}^{n}= & i \sum_{j=0}^{n-1} \int_{t_{j}}^{t_{j+1}} S\left(t_{n}-r\right) V(x)\left(u(r)-u\left(t_{j}\right)\right) \mathrm{d} r \\
= & i \sum_{j=0}^{n-1} \int_{t_{j}}^{t_{j+1}} S\left(t_{n}-r\right) V(x)\left(S\left(r-t_{j}\right)-I\right) u\left(t_{j}\right) \mathrm{d} r \\
& +\sum_{j=0}^{n-1} \int_{t_{j}}^{t_{j+1}} S\left(t_{n}-r\right) V(x)\left(\int_{t_{j}}^{r} S(r-\rho) V(x) u(\rho) \mathrm{d} \rho\right) \mathrm{d} r \\
& +\sum_{j=0}^{n-1} \int_{t_{j}}^{t_{j+1}} S\left(t_{n}-r\right) V(x)\left(\int_{t_{j}}^{r} S(r-\rho) \mathrm{d} W(\rho)\right) \mathrm{d} r \\
= & : J_{1}^{n}+J_{2}^{n}+J_{3}^{n} .
\end{aligned}
$$

Using Lemma 2.1, the fact that $H^{\sigma}$ is an algebra, Hölder's inequality, and Proposition 4.1 we 
obtain

$$
\begin{aligned}
\left\|J_{1}^{n}\right\|_{\sigma} & \leq \sum_{j=0}^{n-1} \int_{t_{j}}^{t_{j+1}}\left\|S\left(t_{n}-r\right) V(x)\left(S\left(r-t_{j}\right)-I\right) u\left(t_{j}\right)\right\|_{\sigma} \mathrm{d} r \\
& \leq C\|V(x)\|_{\sigma} \sum_{j=0}^{n-1} \int_{t_{j}}^{t_{j+1}}\left|r-t_{j}\right|\left\|u\left(t_{j}\right)\right\|_{\sigma+2} \mathrm{~d} r \\
& \leq C k^{2} \sum_{j=0}^{n-1}\left\|u\left(t_{j}\right)\right\|_{\sigma+2} \\
& \leq C k\left(\sup _{0 \leq t \leq T}\|u(t)\|_{\sigma+2}^{2 p}\right)^{\frac{1}{2 p}} \\
& \leq C k
\end{aligned}
$$

so that

$$
\mathbb{E}\left[\max _{n=1, \ldots, N}\left\|J_{1}^{n}\right\|_{\sigma}^{2 p}\right] \leq C k^{2 p}
$$

Next, we estimate $J_{2}^{n}$. Using Lemma 2.1, the fact that $H^{\sigma}$ is an algebra, and Hölder's inequality, we get

$$
\begin{aligned}
\left\|J_{2}^{n}\right\|_{\sigma} & \leq \sum_{j=0}^{n-1} \int_{t_{j}}^{t_{j+1}}\left\|S\left(t_{n}-r\right) V(x)\left(\int_{t_{j}}^{r} S(r-\rho) V(x) u(\rho) \mathrm{d} \rho\right)\right\|_{\sigma} \mathrm{d} r \\
& \leq C\|V(x)\|_{\sigma} \sum_{j=0}^{n-1} \int_{t_{j}}^{t_{j+1}} \int_{t_{j}}^{r}\|V(x)\|_{\sigma}\|u(\rho)\|_{\sigma} \mathrm{d} \rho \mathrm{d} r \\
& \leq C \sum_{j=0}^{n-1} \int_{t_{j}}^{t_{j+1}}\left|r-t_{j}\right|^{\frac{2 p-1}{2 p}}\left(\int_{t_{j}}^{r}\|u(\rho)\|_{\sigma}^{2 p} \mathrm{~d} \rho\right)^{\frac{1}{2 p}} \mathrm{~d} r \\
& \leq C \sum_{j=0}^{n-1} \int_{t_{j}}^{t_{j+1}}\left|r-t_{j}\right|\left(\sup _{t_{j} \leq \rho \leq r}\|u(\rho)\|_{\sigma}^{2 p}\right)^{\frac{1}{2 p}} \mathrm{~d} r \\
& \leq C k\left(\sup _{0 \leq t \leq t_{n}}\|u(t)\|_{\sigma}^{2 p}\right)^{\frac{1}{2 p}}
\end{aligned}
$$

and thus, by Proposition 4.1

$$
\mathbb{E}\left[\max _{n=1, \ldots, N}\left\|J_{2}^{n}\right\|_{\sigma}^{2 p}\right] \leq C k^{2 p} \mathbb{E}\left[\sup _{0 \leq t \leq T}\|u(t)\|_{\sigma}^{2 p}\right] \leq C k^{2 p}
$$

Finally, using Fubini's theorem (when changing the order of integration, we go from $t_{j} \leq r \leq t_{j+1}$ and $t_{j} \leq \rho \leq r$ to $t_{j} \leq \rho \leq t_{j+1}$ and $\left.\rho \leq r \leq t_{j+1}\right)$, the Burkholder inequality, 
Lemma 2.1 and the fact that $H^{\sigma}$ is an algebra, we have

$$
\begin{aligned}
& \mathbb{E}\left[\max _{n=1, \ldots, N}\left\|J_{3}^{n}\right\|_{\sigma}^{2 p}\right]=\mathbb{E}\left[\max _{n=1, \ldots, N}\left\|\sum_{j=0}^{n-1} \int_{t_{j}}^{t_{j+1}} S\left(t_{n}-r\right) V(x)\left(\int_{t_{j}}^{r} S(r-\rho) \mathrm{d} W(\rho)\right) \mathrm{d} r\right\|_{\sigma}^{2 p}\right] \\
& =\mathbb{E}\left[\max _{n=1, \ldots, N}\left\|\sum_{j=0}^{n-1} \int_{t_{j}}^{t_{j+1}} \int_{\rho}^{t_{j+1}} S\left(t_{n}-r\right) V(x) S(r-\rho) \mathrm{d} r \mathrm{~d} W(\rho)\right\|_{\sigma}^{2 p}\right] \\
& =\mathbb{E}\left[\max _{n=1, \ldots, N}\left\|\int_{0}^{t_{n}} \int_{\rho}^{\left[\frac{\rho}{k}+1\right] k} S\left(t_{n}-r\right) V(x) S(r-\rho) \mathrm{d} r \mathrm{~d} W(\rho)\right\|_{\sigma}^{2 p}\right] \\
& \leq \mathbb{E}\left[\sup _{0 \leq t \leq T}\left\|\int_{0}^{t} \int_{\rho}^{\left[\frac{\rho}{k}+1\right] k} S(t-r) V(x) S(r-\rho) \mathrm{d} r \mathrm{~d} W(\rho)\right\|_{\sigma}^{2 p}\right] \\
& \leq C \sup _{0 \leq t \leq T} \mathbb{E}\left[\left\|\int_{0}^{t} \int_{\rho}^{\left[\frac{\rho}{k}+1\right] k} S(t-r) V(x) S(r-\rho) \mathrm{d} r \mathrm{~d} W(\rho)\right\|_{\sigma}^{2 p}\right] \\
& \leq C \sup _{0 \leq t \leq T} \mathbb{E}\left[\left(\int_{0}^{t}\left\|\int_{\rho}^{\left[\frac{\rho}{k}+1\right] k} S(t-r) V(x) S(r-\rho) Q^{1 / 2} \mathrm{~d} r\right\|_{\mathscr{L}_{2}^{\sigma}}^{2} \mathrm{~d} \rho\right)^{p}\right] \\
& =C \max _{n=1, \ldots, N} \mathbb{E}\left[\left(\sum_{j=0}^{n-1} \int_{t_{j}}^{t_{j+1}}\left\|\int_{\rho}^{t_{j+1}} S(t-r) V(x) S(r-\rho) Q^{1 / 2} \mathrm{~d} r\right\|_{\mathscr{L}_{2}^{\sigma}}^{2} \mathrm{~d} \rho\right)^{p}\right] \\
& \leq C \max _{n=1, \ldots, N} \mathbb{E}\left[\left(\sum_{j=0}^{n-1} \int_{t_{j}}^{t_{j+1}}\left(\int_{\rho}^{t_{j+1}}\|V(x)\|_{\sigma}\left\|Q^{1 / 2}\right\|_{\mathscr{L}_{2}^{\sigma}} \mathrm{d} r\right)^{2} \mathrm{~d} \rho\right)^{p}\right] \\
& \leq C \mathbb{E}\left[\left(\sum_{j=0}^{N-1} \int_{t_{j}}^{t_{j+1}}\left|t_{j+1}-\rho\right|^{2} \mathrm{~d} \rho\right)^{p}\right] \\
& \leq C k^{2 p} \text {, }
\end{aligned}
$$

where we recall that $\left[\frac{\rho}{k}+1\right]$ denotes the integer part of $\frac{\rho}{k}+1$, where $k$ is the step size of the scheme.

To summarize, we have obtained the following bound

$$
\mathbb{E}\left[\max _{n=1, \ldots, N}\left\|u^{n}-u\left(t_{n}\right)\right\|_{\sigma}^{2 p}\right] \leq C_{1} k^{2 p}+C_{2} k \sum_{j=0}^{N-1} \mathbb{E}\left[\max _{l=0, \ldots, j}\left\|u^{l}-u\left(t_{l}\right)\right\|_{\sigma}^{2 p}\right]
$$

and an application of Gronwall's lemma completes the proof. $\square$

4.2. A trace formula for the mass. With appropriate boundary conditions, such as when the domain is the $d$-dimensional torus $\mathbb{T}^{d}$, the expected mass

$$
\mathbb{E}[M(u)]=\mathbb{E}\left[\int_{\mathbb{T}^{d}}|u|^{2} \mathrm{~d} x\right]
$$

still exhibits linear growth. On a smooth compact Riemannian manifold $(\widetilde{M}, g)$, such as the $d$-dimensional torus, we still have $H^{2}(\widetilde{M}) \subset L^{\infty}(\widetilde{M})$ if $d=1,2,3$ (see [21, Theorem 2.7]). In particular, Propositions 4.1 and 4.2 and Theorem 4.3 above remain valid when $\mathbb{R}^{d}$ is replaced by $\mathbb{T}^{d}$. Therefore, in this subsection, we assume that the spatial domain is the $d$-dimensional torus $\mathbb{T}^{d}$.

PROPOSITION 4.5. Let $d=1,2,3$ and assume that the initial data is such that $\mathbb{E}\left[M\left(u_{0}\right)\right]$ is finite, that $V(x) \in H^{2}$, and that $Q$ is trace-class. Then the exact solution of 44.1, given by 
equation (4.2), satisfies the trace formula

$$
\mathbb{E}[M(u(t))]=\mathbb{E}\left[M\left(u_{0}\right)\right]+t \operatorname{Tr}(Q),
$$

for $t \geq 0$.

Proof. This follows from the Ito formula [11, Theorem 4.17]. We have

$$
\begin{aligned}
M(u(t))= & M\left(u_{0}\right)+\int_{0}^{t}\left(M^{\prime}(u(s)), \Phi(s) \mathrm{d} W(s)\right)+\int_{0}^{t}\left(M^{\prime}(u(s)), \varphi(s)\right) \mathrm{d} s \\
& +\int_{0}^{t} \frac{1}{2} \operatorname{Tr}\left[M^{\prime \prime}(u(s))\left(\Phi(s) Q^{1 / 2}\right)\left(\Phi(s) Q^{1 / 2}\right)^{*}\right] \mathrm{d} s,
\end{aligned}
$$

where $\varphi(s)=-\mathrm{i} \Delta u(s)-\mathrm{i} V(x) u(s)$ and $\Phi(s)=I$. The expected value of the first integral on the right-hand side is zero. For the second integral, we have

$$
\begin{aligned}
\left(M^{\prime}(u(s)), \varphi(s)\right)= & (u(s), \varphi(s))+(\varphi(s), u(s)) \\
= & (u(s),-\mathrm{i} \Delta u(s))+(u(s),-\mathrm{i} V(x) u(s)) \\
& +(-\mathrm{i} \Delta u(s), u(s))+(-\mathrm{i} V(x) u(s), u(s)) \\
= & 0 .
\end{aligned}
$$

Thus, we arrive at

$$
\begin{aligned}
\mathbb{E}[M(u(t))] & =\mathbb{E}\left[M\left(u_{0}\right)\right]+\frac{1}{2} \int_{0}^{t} \mathbb{E}\left[\operatorname{Tr}\left(M^{\prime \prime}(u(s))\left(Q^{1 / 2}\right)\left(Q^{1 / 2}\right)^{*}\right)\right] \mathrm{d} s \\
& =\mathbb{E}\left[M\left(u_{0}\right)\right]+t \operatorname{Tr}(Q) .
\end{aligned}
$$

We now investigate how the expected mass of our exponential integrator behaves. As seen below we do not get an exact trace formula as for the linear problem considered in Section 3, but instead we get a trace formula with an error of size $\mathscr{O}(k)$ on compact intervals.

Proposition 4.6. Let $d=1,2,3$ and assume that the initial data is such that $\mathbb{E}\left[M\left(u_{0}\right)\right]$ is finite, that $V(x) \in H^{2}$, and that $Q$ is trace-class. Then the stochastic exponential integrator $u^{n}$ given by (4.3) satisfies the following almost trace formula for the expected mass

$$
\mathbb{E}\left[M\left(u^{n}\right)\right]=\mathbb{E}\left[M\left(u_{0}\right)\right]+t_{n} \operatorname{Tr}(Q)+\mathscr{O}(k) \quad \text { for } \quad 0 \leq t_{n}=n k \leq T .
$$

Proof. First note that, by assumption, we have $\mathbb{E}\left[\left\|u_{0}\right\|_{L^{2}}^{2}\right]<\infty$.

The numerical method reads

$$
u^{n}=S(k) u^{n-1}-\mathrm{i} S(k) V(x) u^{n-1} k-\mathrm{i} \int_{t_{n-1}}^{t_{n}} S(k) \mathrm{d} W(r)
$$

and a straight-forward calculation gives

$$
\begin{aligned}
\left\|u^{n}\right\|_{L^{2}}^{2}= & \left\|S(k) u^{n-1}\right\|_{L^{2}}^{2}+\left(S(k) u^{n-1},-\mathrm{i} S(k) V(x) u^{n-1} k\right) \\
& +\left(S(k) u^{n-1},-\mathrm{i} \int_{t_{n-1}}^{t_{n}} S(k) \mathrm{d} W(r)\right)+\left(-\mathrm{i} S(k) V(x) u^{n-1} k, S(k) u^{n-1}\right) \\
& +\left\|\mathrm{i} S(k) V(x) u^{n-1} k\right\|_{L^{2}}^{2}+\left(-\mathrm{i} S(k) V(x) u^{n-1} k,-\mathrm{i} \int_{t_{n-1}}^{t_{n}} S(k) \mathrm{d} W(r)\right) \\
& +\left(-\mathrm{i} \int_{t_{n-1}}^{t_{n}} S(k) \mathrm{d} W(r), S(k) u^{n-1}\right)+\left(-\mathrm{i} \int_{t_{n-1}}^{t_{n}} S(k) \mathrm{d} W(r),-\mathrm{i} S(k) V(x) u^{n-1} k\right) \\
& +\left\|\mathrm{i} \int_{t_{n-1}}^{t_{n}} S(k) \mathrm{d} W(r)\right\|_{L^{2}}^{2} .
\end{aligned}
$$


Next we observe that $\left(S(k) u^{n-1},-\mathrm{i} S(k) V(x) u^{n-1} k\right)+\left(-\mathrm{i} S(k) V(x) u^{n-1} k, S(k) u^{n-1}\right)=0$ and the expected value of the terms containing one stochastic integral is zero. We thus have, using Lemma 2.1. Proposition 4.2 (case (ii)), and Ito's isometry,

$$
\begin{aligned}
\mathbb{E}\left[\left\|u^{n}\right\|_{L^{2}}^{2}\right] & =\mathbb{E}\left[\left\|u^{n-1}\right\|_{L^{2}}^{2}\right]+k^{2} \mathbb{E}\left[\left\|V(x) u^{n-1}\right\|_{L^{2}}^{2}\right]+\mathbb{E}\left[\left\|\int_{t_{n-1}}^{t_{n}} S(k) \mathrm{d} W(r)\right\|_{L^{2}}^{2}\right] \\
& =\mathbb{E}\left[M\left(u^{n-1}\right)\right]+C k^{2}+\int_{t_{n-1}}^{t_{n}}\left\|Q^{1 / 2}\right\|_{\mathscr{L}_{2}^{0}}^{2} \mathrm{~d} r \\
& =\mathbb{E}\left[M\left(u^{n-1}\right)\right]+C k^{2}+k \operatorname{Tr}(Q) .
\end{aligned}
$$

An iteration completes the proof.

4.3. A trace formula for the energy. As for the mass, with suitable boundary conditions, the expected energy will still grow linearly. We again consider the domain to be the $d$-dimensional torus and the energy is given by

$$
H(u)=\frac{1}{2} \int_{\mathbb{T}^{d}}|\nabla u|^{2} \mathrm{~d} x-\frac{1}{2} \int_{\mathbb{T}^{d}} V(x)|u|^{2} \mathrm{~d} x .
$$

Proposition 4.7. Let $d=1,2,3$ and assume that the initial data is such that $\mathbb{E}\left[H\left(u_{0}\right)\right]$ is finite, and that $Q^{1 / 2} \in \mathscr{L}_{2}^{1}$. If $d=1$, we assume $V(x) \in H^{2}$ and if $d=2,3$ we assume $V(x) \in H^{3}$. Then the exact solution of 4.1], given by equation [4.2), satisfies the trace formula

$$
\mathbb{E}[H(u(t))]=\mathbb{E}\left[H\left(u_{0}\right)\right]+\frac{t}{2}\left(\operatorname{Tr}(\nabla Q \nabla)-\operatorname{Tr}\left(Q^{1 / 2} V(x) Q^{1 / 2}\right)\right)
$$

Proof. Similar to the trace formula for the mass, we use the Ito formula

$$
\begin{aligned}
H(u(t))= & H\left(u_{0}\right)+\int_{0}^{t}\left(H^{\prime}(u(s)), \Phi(s) \mathrm{d} W(s)\right)+\int_{0}^{t}\left(H^{\prime}(u(s)), \varphi(s)\right) \mathrm{d} s \\
& +\int_{0}^{t} \frac{1}{2} \operatorname{Tr}\left[H^{\prime \prime}(u(s))\left(\Phi(s) Q^{1 / 2}\right)\left(\Phi(s) Q^{1 / 2}\right)^{*}\right] \mathrm{d} s,
\end{aligned}
$$

where $\varphi(s)=-\mathrm{i} \Delta u(s)-\mathrm{i} V(x) u(s)$ and $\Phi(s)=I$. The expected value of the first integral is seen to be zero, and a similar calculation as in the proof of Proposition 4.5 shows that the second integral is zero as well. To calculate the third integral, we note that

$$
\begin{aligned}
\operatorname{Tr}\left(H^{\prime \prime}(u(s))\left(Q^{1 / 2}\right)\left(Q^{1 / 2}\right)^{*}\right) & =\sum_{n \geq 1} H^{\prime \prime}(u(s))\left(Q^{1 / 2} e_{n}, Q^{1 / 2} e_{n}\right) \\
& =\sum_{n \geq 1}\left(\nabla Q^{1 / 2} e_{n}, \nabla Q^{1 / 2} e_{n}\right)_{L^{2}}-\sum_{n \geq 1}\left(V(x) Q^{1 / 2} e_{n}, Q^{1 / 2} e_{n}\right)_{L^{2}} \\
& =\operatorname{Tr}(\nabla Q \nabla)-\operatorname{Tr}\left(Q^{1 / 2} V(x) Q^{1 / 2}\right) .
\end{aligned}
$$

The energy for the numerical approximation satisfies an almost trace formula: we get a small error of size $\mathscr{O}(k)$, as seen in the next proposition.

Proposition 4.8. Let $d=1,2,3$ and assume that $\mathbb{E}\left[\left\|u_{0}\right\|_{2}^{2}\right] \leq C$. If $d=1$ then assume $V(x) \in H^{2}$. If $d=2,3$ then assume $V(x) \in H^{3}$. Also assume that $Q^{1 / 2} \in \mathscr{L}_{2}^{2}$. Then the stochastic exponential integrator $u^{n}$ given by (3.2) satisfies the following almost trace formula for the expected energy

$$
\mathbb{E}\left[H\left(u^{n}\right)\right]=\mathbb{E}\left[H\left(u_{0}\right)\right]+\frac{t_{n}}{2}\left(\operatorname{Tr}(\nabla Q \nabla)-\operatorname{Tr}\left(Q^{1 / 2} V(x) Q^{1 / 2}\right)\right)+\mathscr{O}(k) \quad \text { for } \quad 0 \leq t_{n}=n k \leq T .
$$


Proof. First we note that with these assumptions we have finite initial energy, since

$$
\begin{aligned}
\mathbb{E}\left[H\left(u_{0}\right)\right] & =\mathbb{E}\left[\left\|\nabla u_{0}\right\|_{L^{2}}^{2}\right]-\mathbb{E}\left[\int_{\mathbb{T}^{d}} V(x)\left|u_{0}\right|^{2} \mathrm{~d} x\right] \\
& \leq \mathbb{E}\left[\left\|u_{0}\right\|_{1}^{2}\right]+\|V(x)\|_{L^{\infty}}^{2} \mathbb{E}\left[\left\|u_{0}\right\|_{L^{2}}^{2}\right] \\
& \leq C .
\end{aligned}
$$

We add and subtract the energy for the exact solution

$$
\begin{aligned}
\mathbb{E}\left[H\left(u^{n}\right)\right] & =\mathbb{E}\left[H\left(u\left(t_{n}\right)\right)\right]+\mathbb{E}\left[H\left(u^{n}\right)-H\left(u\left(t_{n}\right)\right)\right] \\
& =\mathbb{E}\left[H\left(u_{0}\right)\right]+\frac{t_{n}}{2}\left(\operatorname{Tr}(\nabla Q \nabla)-\operatorname{Tr}\left(Q^{1 / 2} V(x) Q^{1 / 2}\right)\right)+\mathbb{E}\left[H\left(u^{n}\right)-H\left(u\left(t_{n}\right)\right)\right] .
\end{aligned}
$$

Thus we need to show that $\left|\mathbb{E}\left[H\left(u^{n}\right)-H\left(u\left(t_{n}\right)\right)\right]\right| \leq C k$. We have (omitting the constant $1 / 2$ for ease of presentation)

$$
\begin{aligned}
\left|\mathbb{E}\left[H\left(u^{n}\right)-H\left(u\left(t_{n}\right)\right)\right]\right| \leq & \left|\mathbb{E}\left[\left\|\nabla u^{n}\right\|_{L^{2}}^{2}-\left\|\nabla u\left(t_{n}\right)\right\|_{L^{2}}^{2}\right]\right| \\
& +\left|\mathbb{E}\left[\int_{\mathbb{T}^{d}} V(x)\left|u\left(t_{n}\right)\right|^{2} \mathrm{~d} x-\int_{\mathbb{T}^{d}} V(x)\left|u^{n}\right|^{2} \mathrm{~d} x\right]\right| \\
= & : I_{1}^{n}+I_{2}^{n} .
\end{aligned}
$$

We begin by estimating $I_{1}^{n}$. We have, using that the inner product defined by (2.2) is symmetric, integration by parts and the boundary conditions, Cauchy-Schwarz and Hölder's inequalities,

$$
\begin{aligned}
I_{1}^{n} & =\left|\mathbb{E}\left[\left(\nabla\left(u^{n}+u\left(t_{n}\right)\right), \nabla\left(u^{n}-u\left(t_{n}\right)\right)\right)\right]\right| \\
& =\left|\mathbb{E}\left[\left(\Delta\left(u^{n}+u\left(t_{n}\right)\right), u^{n}-u\left(t_{n}\right)\right)\right]\right| \\
& \leq \mathbb{E}\left[\left\|\Delta\left(u^{n}+u\left(t_{n}\right)\right)\right\|_{L^{2}}\left\|u^{n}-u\left(t_{n}\right)\right\|_{L^{2}}\right] \\
& \leq\left(\mathbb{E}\left[\left\|\Delta\left(u^{n}+u\left(t_{n}\right)\right)\right\|_{L^{2}}^{2}\right]\right)^{1 / 2}\left(\mathbb{E}\left[\left\|u^{n}-u\left(t_{n}\right)\right\|_{L^{2}}^{2}\right]\right)^{1 / 2} \\
& \leq\left(\mathbb{E}\left[\left\|u^{n}+u\left(t_{n}\right)\right\|_{2}^{2}\right]\right)^{1 / 2}\left(\mathbb{E}\left[\left\|u^{n}-u\left(t_{n}\right)\right\|_{L^{2}}^{2}\right]\right)^{1 / 2} .
\end{aligned}
$$

Using Propositions 4.1,4.2, and Theorem 4.3 we have

$$
I_{1}^{n} \leq C k
$$

Similarly, we have

$$
\begin{aligned}
I_{2}^{n} & =\left|\mathbb{E}\left[\int_{\mathbb{T}^{d}} V(x)\left(\left|u\left(t_{n}\right)\right|^{2}-\left|u^{n}\right|^{2}\right) \mathrm{d} x\right]\right| \\
& \leq\|V(x)\|_{L^{\infty}}\left|\mathbb{E}\left[\left\|u\left(t_{n}\right)\right\|_{L^{2}}^{2}-\left\|u^{n}\right\|_{L^{2}}^{2}\right]\right| \\
& \leq C \mathbb{E}\left[\left\|u\left(t_{n}\right)+u^{n}\right\|_{L^{2}}\left\|u\left(t_{n}\right)-u^{n}\right\|_{L^{2}}\right] \\
& \leq C\left(\mathbb{E}\left[\left\|u\left(t_{n}\right)+u^{n}\right\|_{L^{2}}^{2}\right]\right)^{1 / 2}\left(\mathbb{E}\left[\left\|u\left(t_{n}\right)-u^{n}\right\|_{L^{2}}^{2}\right]\right)^{1 / 2} \\
& \leq C k .
\end{aligned}
$$

( 


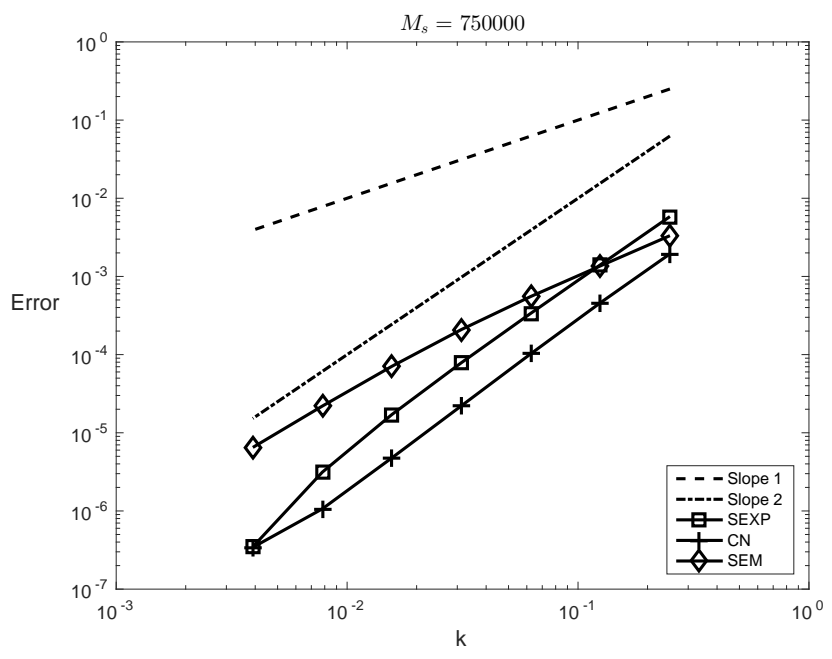

FIGURE 4.1. Stochastic Schrödinger equation with potential: Mean-square errors for the stochastic exponential integrator (SEXP), the Crank-Nicolson scheme $(C N)$, and the semi-implicit Euler-Maruyama (SEM). The dotted, resp. dash-dotted, lines have slopes 1 and 2.

4.4. Numerical experiments for the stochastic Schrödinger equations with a multiplicative potential. This subsection illustrates some of the above properties (error estimates and trace formula for the mass) of the stochastic exponential integrator (4.3) when applied to the linear stochastic Schrödinger equation with a multiplicative potential on the interval $[0,2 \pi]$ with periodic boundary conditions 44.1 . In these numerical experiments, we consider a potential $V(x)=\frac{1}{1+\sin ^{2}(x)}$, [4], and initial values with $u_{0}(x)=\frac{2}{2-\cos (x)}$. We compare the stochastic exponential integrator (SEXP) with the Crank-Nicolson scheme $(\mathrm{CN})$ and the semi-implicit Euler-Maruyama scheme, explicit in $V(x) u$, (SEM).

Figure 4.1 illustrates the convergence errors of the above numerical methods for a noise with covariance operator having the eigenvalues $\lambda_{n}=1 /\left(1+n^{6}\right)$ for $n \in \mathbb{Z}$. The spatial discretisation is done by a pseudospectral method with $M=2^{8}$ Fourier modes. The rates of mean-square convergence (measured in the $L^{2}$-norm at the end of the interval of integration $[0,0.5])$ of these numerical methods are presented in this figure. The expected rate of convergence $\mathscr{O}\left(k^{2}\right)$ of the stochastic exponential integrator, as stated in Theorem 4.3, can be confirmed. Here, the exact solution is approximated by the stochastic exponential method with a very small time step $k_{\text {exact }}=2^{-9}$ and $M_{\mathrm{s}}=750000$ samples are used for the approximation of the expected values.

We now consider eigenvalues of $Q$ given by $\lambda_{n}=1 /\left(1+n^{2}\right)$ and examine the numerical trace formulas for the mass. Figure 4.2 is produced using $M=2^{7}$ Fourier modes for the spatial discretisation; $M_{\mathrm{S}}=10000$ samples for the approximatation of the expected values; the time interval is $[0,5]$; and a step size $k=0.1$. As stated by Proposition 4.6 in this figure, one can observe the small error in the preservation of the trace formula for the mass of the numerical solution given by the exponential integrator.

5. Stochastic Schrödinger equations driven by multiplicative noise. We continue our exposition of the exponential integrator applied to the stochastic Schrödinger equation by considering the equation driven by a multiplicative Ito noise

$$
\begin{array}{ll}
\operatorname{id} u=\Delta u \mathrm{~d} t+V(x) u \mathrm{~d} t+u \mathrm{~d} W & \text { in } \mathbb{R}^{d} \times(0, \infty), \\
u(\cdot, 0)=u_{0} & \text { in } \mathbb{R}^{d} .
\end{array}
$$




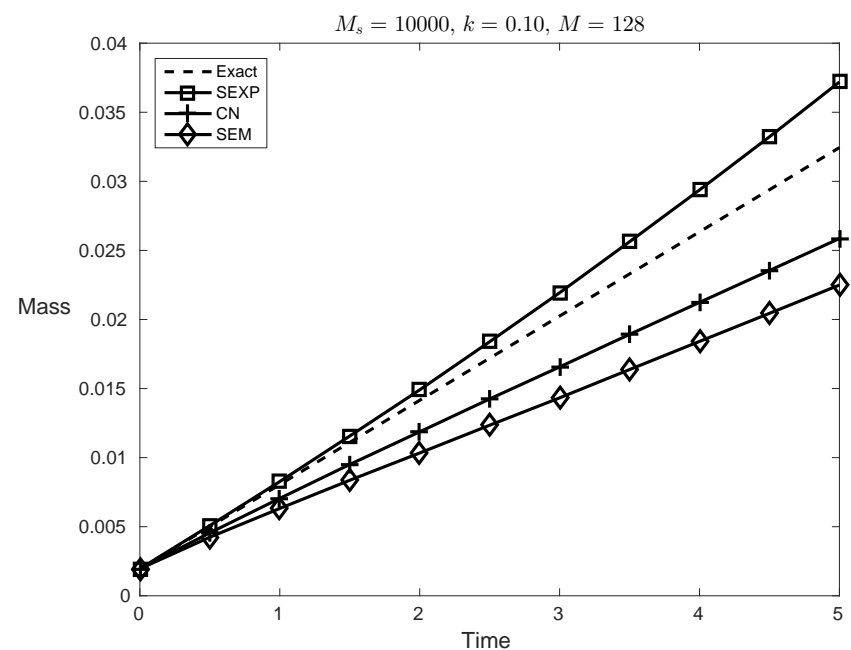

FIGURE 4.2. Stochastic Schrödinger equation with potential: Mass trace formulas for the numerical solutions given by the stochastic exponential method (SEXP), the Crank-Nicolson scheme (CN) and the semi-implicit EulerMaruyama scheme (SEM). Time interval and time step: [0,5], resp. $k=0.1$.

As before, $V(x)$ is a real-valued potential, $u_{0}$ is an $\mathscr{F}_{0}$-measurable complex-valued random variable, and the $Q$-Wiener process is complex-valued and square integrable. Further assumptions on $d, u_{0}, V(x)$, and $Q$ will be made in the results of this section. Again, the equation can be written in its mild form

$$
u(t)=S(t) u_{0}-\mathrm{i} \int_{0}^{t} S(t-r) V(x) u(r) \mathrm{d} r-\mathrm{i} \int_{0}^{t} S(t-r) u(r) \mathrm{d} W(r),
$$

where $S(t)=\mathrm{e}^{-\mathrm{i} t \Delta}$.

Proposition 5.1. Let $\sigma>\frac{d}{2}$, and assume that $\mathbb{E}\left[\left\|u_{0}\right\|_{\sigma}^{2 p}\right]<\infty$ for some $p \in \mathbb{N}, V(x) \in$ $H^{\sigma}$, and $Q^{1 / 2} \in \mathscr{L}_{2}^{\sigma}$. Then there exists a unique solution $u(t)$ on $[0, T]$, for some $T>0$, to problem 5.1] which satisfies

$$
\mathbb{E}\left[\sup _{0 \leq t \leq T}\|u(t)\|_{\sigma}^{2 p}\right] \leq C
$$

REMARK 5.2. The result of Proposition 5.1 holds with $\sigma=0$ if we assume $d=1,2,3$, $\mathbb{E}\left[\left\|u_{0}\right\|_{0}^{2 p}\right]<\infty$ for some $p \in \mathbb{N}, V(x) \in H^{2}$, and $Q^{1 / 2} \in \mathscr{L}_{2}^{2}$.

Proof. The existence and uniqueness of a solution again follows by a standard fixed point argument as used in [11, Theorem 7.4]. From the mild equation and the proof of Proposition 
4.1 we have

$$
\begin{aligned}
\mathbb{E}\left[\sup _{0 \leq t \leq T}\|u(t)\|_{\sigma}^{2 p}\right] \leq & C\left(\mathbb{E}\left[\sup _{0 \leq t \leq T}\left\|S(t) u_{0}\right\|_{\sigma}^{2 p}\right]\right. \\
& +\mathbb{E}\left[\sup _{0 \leq t \leq T}\left\|\int_{0}^{t} S(t-r) V(x) u(r) \mathrm{d} r\right\|_{\sigma}^{2 p}\right] \\
& \left.+\mathbb{E}\left[\sup _{0 \leq t \leq T}\left\|\int_{0}^{t} S(t-r) u(r) \mathrm{d} W(r)\right\|_{\sigma}^{2 p}\right]\right) \\
\leq & C_{1}+C_{2} \int_{0}^{T} \mathbb{E}\left[\sup _{0 \leq s \leq t}\|u(s)\|_{\sigma}^{2 p}\right] \mathrm{d} t \\
& +C_{3} \mathbb{E}\left[\sup _{0 \leq t \leq T}\left\|\int_{0}^{t} S(t-r) u(r) \mathrm{d} W(r)\right\|_{\sigma}^{2 p}\right] .
\end{aligned}
$$

We need to bound the stochastic integral. Using the Burkholder inequality, that $H^{\sigma}$ forms an algebra, and Hölder's inequality, we have

$$
\begin{aligned}
\mathbb{E}\left[\sup _{0 \leq t \leq T}\left\|\int_{0}^{t} S(t-r) u(r) \mathrm{d} W(r)\right\|_{\sigma}^{2 p}\right] & \leq C \mathbb{E}\left[\left(\int_{0}^{T}\left\|u(t) Q^{1 / 2}\right\|_{\mathscr{L}_{2}^{\sigma}}^{2} \mathrm{~d} t\right)^{p}\right] \\
& \leq C \mathbb{E}\left[\left(\int_{0}^{T}\|u(t)\|_{\sigma}^{2}\left\|Q^{1 / 2}\right\|_{\mathscr{L}_{2}^{\sigma}}^{2} \mathrm{~d} t\right)^{p}\right] \\
& \leq C \mathbb{E}\left[\int_{0}^{T}\|u(t)\|_{\sigma}^{2 p} \mathrm{~d} t\right] \\
& \leq C \int_{0}^{T} \mathbb{E}\left[\sup _{0 \leq s \leq t}\|u(s)\|_{\sigma}^{2 p}\right] \mathrm{d} t .
\end{aligned}
$$

The result follows from Gronwall's lemma.

We will need the following lemma regarding regularity of the exact solution.

Lemma 5.3. Let $\sigma>\frac{d}{2}$ and assume $\mathbb{E}\left[\left\|u_{0}\right\|_{\sigma+1}^{2 p}\right]<\infty$ for some $p \in \mathbb{N}, V(x) \in H^{\sigma+1}$, and $Q^{1 / 2} \in \mathscr{L}_{2}^{\sigma+1}$. Then, for $0 \leq s \leq t<\infty$, the exact solution of (5.1) satisfies the following regularity estimate

$$
\mathbb{E}\left[\|u(t)-u(s)\|_{\sigma}^{2 p}\right] \leq C|t-s|^{p} .
$$

REMARK 5.4. The result of Lemma 5.3 holds with $\sigma=0$ if we assume $d=1,2,3$, $\mathbb{E}\left[\left\|u_{0}\right\|_{2}^{2 p}\right]<\infty$ for some $p \in \mathbb{N}, V(x) \in H^{2}$, and $Q^{1 / 2} \in \mathscr{L}_{2}^{2}$.

Proof. Using the mild equation, we have

$$
\begin{aligned}
u(t)-u(s)= & (S(t-s)-I) u(s)-\mathrm{i} \int_{s}^{t} S(t-r) V(x) u(r) \mathrm{d} r \\
& -\mathrm{i} \int_{s}^{t} S(t-r) u(r) \mathrm{d} W(r) .
\end{aligned}
$$

We proceed to estimate the three terms on the right hand side. The first one, due to Lemma 2.2 reads

$$
\begin{aligned}
\|(S(t-s)-I) u(s)\|_{\sigma} & \leq\|S(t-s)-I\|_{\mathscr{L}\left(H^{\sigma+1}, H^{\sigma}\right)}\|u(s)\|_{\sigma+1} \\
& \leq C|t-s|^{1 / 2}\|u(s)\|_{\sigma+1},
\end{aligned}
$$


so that, by Proposition 5.1

$$
\mathbb{E}\left[\|(S(t-s)-I) u(s)\|_{\sigma}^{2 p}\right] \leq C|t-s|^{p} .
$$

To estimate the second term, we use that $H^{\sigma}$ forms an algebra and Hölder's inequality

$$
\begin{aligned}
\left\|\int_{s}^{t} S(t-r) V(x) u(r) \mathrm{d} r\right\|_{\sigma} & \leq \int_{s}^{t}\|V(x) u(r)\|_{\sigma} \mathrm{d} r \\
& \leq C \int_{s}^{t}\|V(x)\|_{\sigma}\|u(r)\|_{\sigma} \mathrm{d} r \\
& \leq C|t-s|\left(\sup _{s \leq r \leq t}\|u(r)\|_{\sigma}^{2 p}\right)^{\frac{1}{2 p}} .
\end{aligned}
$$

Taking expectation of the $2 p$-th power of the above expression and using Proposition 5.1 we get

$$
\mathbb{E}\left[\left\|\int_{s}^{t} S(t-r) V(x) u(r) \mathrm{d} r\right\|_{\sigma}^{2 p}\right] \leq C|t-s|^{2 p} .
$$

Finally, we have

$$
\begin{aligned}
\mathbb{E}\left[\left\|\int_{s}^{t} S(t-r) u(r) \mathrm{d} W(r)\right\|_{\sigma}^{2 p}\right] & \leq C \mathbb{E}\left[\left(\int_{s}^{t}\left\|u(r) Q^{1 / 2}\right\|_{\mathscr{L}_{2}^{\sigma}}^{2} \mathrm{~d} r\right)^{p}\right] \\
& \leq C|t-s|^{p} \mathbb{E}\left[\sup _{s \leq r \leq t}\|u(r)\|_{\sigma}^{2 p}\right] \\
& \leq C|t-s|^{p} .
\end{aligned}
$$

5.1. Error analysis for multiplicative noise. As before, we let $N>0$ be an integer and divide the interval $[0, T]$ into subintervals $0=t_{0}<t_{1}<\ldots<t_{N-1}<t_{N}=T$ of equal length $k$ so that $t_{n}=n k$. We discretise the mild equation and our exponential integrator now reads

$$
\begin{aligned}
u^{n} & =S(k) u^{n-1}-\mathrm{i} k S(k) V(x) u^{n-1}-\mathrm{i} S(k) u^{n-1} \Delta W^{n-1} \\
& =S\left(t_{n}\right) u_{0}-\mathrm{i} \sum_{j=0}^{n-1} \int_{t_{j}}^{t_{j+1}} S\left(t_{n}-t_{j}\right) V(x) u^{j} \mathrm{~d} r-\mathrm{i} \sum_{j=0}^{n-1} \int_{t_{j}}^{t_{j+1}} S\left(t_{n}-t_{j}\right) u^{j} \mathrm{~d} W(r),
\end{aligned}
$$

where $\Delta W^{n-1}=W\left(t_{n}\right)-W\left(t_{n-1}\right)$.

As seen in the theorem below, because of the multiplicative noise, the order of convergence is reduced to one half.

THEOREM 5.5. Consider the time integration of the stochastic Schrödinger equation (5.1) given by the exponential integrator [5.2). Let $\sigma>\frac{d}{2}$ and assume that $\mathbb{E}\left[\left\|u_{0}\right\|_{\sigma+1}^{2 p}\right]<\infty$ for some $p \in \mathbb{N}$. Assume also that $V(x) \in H^{\sigma+1}$ and $Q^{1 / 2} \in \mathscr{L}_{2}^{\sigma+1}$. Then there exists a constant $C$ such that

$$
\mathbb{E}\left[\max _{n=1, \ldots N}\left\|u^{n}-u\left(t_{n}\right)\right\|_{\sigma}^{2 p}\right] \leq C k^{p}
$$

REMARK 5.6. Following remarks 5.2] and 5.4 the result of Theorem 5.5 holds with $\sigma=0$ if $d=1,2,3, \mathbb{E}\left[\left\|u_{0}\right\|_{2}^{2 p}\right]<\infty$ for some $p \in \mathbb{N}, V(x) \in H^{2}$, and $Q^{1 / 2} \in \mathscr{L}_{2}^{2}$. 
REMARK 5.7. We could replace the nonlinear term and the $u$ in front of the noise in equation (5.1) with more general bounded Lipschitz functions $F: H^{\sigma} \rightarrow H^{\sigma}$ and $\Phi: H^{\sigma} \rightarrow$ $\mathscr{L}_{2}^{\sigma}$. The above result holds with similar assumptions as in Hypothesis 5.1 and 5.2 in [17], that is if we assume that the solution $(u(t))_{t \in[0, T]}$ is in $L^{2 p}\left(\Omega ; L^{\infty}\left(0, T ; H^{\sigma+1}\right),(F(u(t)))_{t \in[0, T]}\right.$ is in $L^{2 p}\left(\Omega ; L^{\infty}\left(0, T ; H^{\sigma+1}\right)\right),(\Phi(u(t)))_{t \in[0, T]}$ is in $L^{2 p}\left(\Omega ; L^{\infty}\left(0, T ; \mathscr{L}_{2}^{\sigma+1}\right)\right)$, and $u_{0} \in L^{2 p}\left(\Omega ; H^{\sigma+1}\right)$.

Proof. Using the mild formulation, we have

$$
\begin{aligned}
u^{n}-u\left(t_{n}\right)= & \mathrm{i} \sum_{j=0}^{n-1} \int_{t_{j}}^{t_{j+1}}\left(S\left(t_{n}-r\right) V(x) u(r)-S\left(t_{n}-t_{j}\right) V(x) u^{j}\right) \mathrm{d} r \\
& +\mathrm{i} \sum_{j=0}^{n-1} \int_{t_{j}}^{t_{j+1}}\left(S\left(t_{n}-r\right) u(r)-S\left(t_{n}-t_{j}\right) u^{j}\right) \mathrm{d} W(r) \\
= & : \operatorname{Err}_{V}^{n}+\operatorname{Err}_{W}^{n} .
\end{aligned}
$$

We add and subtract suitable terms to get

$$
\begin{aligned}
\operatorname{Err}_{V}^{n}= & \mathrm{i} \sum_{j=0}^{n-1} \int_{t_{j}}^{t_{j+1}} S\left(t_{n}-r\right) V(x)\left(u(r)-u\left(t_{j}\right)\right) \mathrm{d} r \\
& +\mathrm{i} \sum_{j=0}^{n-1} \int_{t_{j}}^{t_{j+1}}\left(S\left(t_{n}-r\right)-S\left(t_{n}-t_{j}\right)\right) V(x) u\left(t_{j}\right) \mathrm{d} r \\
& +\mathrm{i} \sum_{j=0}^{n-1} \int_{t_{j}}^{t_{j+1}} S\left(t_{n}-t_{j}\right) V(x)\left(u\left(t_{j}\right)-u^{j}\right) \mathrm{d} r \\
= & : I_{1}^{n}+I_{2}^{n}+I_{3}^{n},
\end{aligned}
$$

and

$$
\begin{aligned}
\operatorname{Err}_{W}^{n}= & \mathrm{i} \sum_{j=0}^{n-1} \int_{t_{j}}^{t_{j+1}} S\left(t_{n}-r\right)\left(u(r)-u\left(t_{j}\right)\right) \mathrm{d} W(r) \\
& +\mathrm{i} \sum_{j=0}^{n-1} \int_{t_{j}}^{t_{j+1}}\left(S\left(t_{n}-r\right)-S\left(t_{n}-t_{j}\right)\right) u\left(t_{j}\right) \mathrm{d} W(r) \\
& +\mathrm{i} \sum_{j=0}^{n-1} \int_{t_{j}}^{t_{j+1}} S\left(t_{n}-t_{j}\right)\left(u\left(t_{j}\right)-u^{j}\right) \mathrm{d} W(r) \\
= & : J_{1}^{n}+J_{2}^{n}+J_{3}^{n} .
\end{aligned}
$$

We now proceed to estimate each of these terms. For $I_{1}^{n}$, using again that $[r / k]$ denotes the integer part of $r / k$ and then Hölder's inequality, we have

$$
\begin{aligned}
\left\|I_{1}^{n}\right\|_{\sigma} & \leq \sum_{j=0}^{n-1} \int_{t_{j}}^{t_{j+1}}\left\|V(x)\left(u(r)-u\left(t_{j}\right)\right)\right\|_{\sigma} \mathrm{d} r \\
& \leq C\|V(x)\|_{\sigma} \sum_{j=0}^{n-1} \int_{t_{j}}^{t_{j+1}}\left\|u(r)-u\left(t_{j}\right)\right\|_{\sigma} \mathrm{d} r \\
& \leq C \int_{0}^{t_{n}}\|u(r)-u([r / k] k)\|_{\sigma} \mathrm{d} r \\
& \leq C T^{\frac{2 p-1}{2 p}}\left(\int_{0}^{t_{n}}\|u(r)-u([r / k] k)\|_{\sigma}^{2 p} \mathrm{~d} r\right)^{\frac{1}{2 p}}
\end{aligned}
$$


so that, using Lemma 5.3 .

$$
\begin{aligned}
\mathbb{E}\left[\max _{n=1, \ldots, N}\left\|I_{1}^{n}\right\|_{\sigma}^{2 p}\right] & \leq C \mathbb{E}\left[\int_{0}^{T}\|u(r)-u([r / k] k)\|_{\sigma}^{2 p} \mathrm{~d} r\right] \\
& \leq C \sum_{j=0}^{N-1} \int_{t_{j}}^{t_{j+1}} \mathbb{E}\left[\left\|u(r)-u\left(t_{j}\right)\right\|_{\sigma}^{2 p}\right] \mathrm{d} r \\
& \leq C \sum_{j=0}^{N-1} \int_{t_{j}}^{t_{j+1}}\left|r-t_{j}\right|^{p} \mathrm{~d} r \\
& \leq C k^{p} .
\end{aligned}
$$

By Lemma2.2 and Hölder's inequality, we have

$$
\begin{aligned}
\left\|I_{2}^{n}\right\|_{\sigma} & \leq \sum_{j=0}^{n-1} \int_{t_{j}}^{t_{j+1}}\left\|S\left(t_{n}-t_{j}\right)\left(S\left(t_{j}-r\right)-I\right) V(x) u\left(t_{j}\right)\right\|_{\sigma} \mathrm{d} r \\
& \leq C \sum_{j=0}^{n-1} \int_{t_{j}}^{t_{j+1}}\left|t_{j}-r\right|^{1 / 2}\|V(x)\|_{\sigma+1}\left\|u\left(t_{j}\right)\right\|_{\sigma+1} \mathrm{~d} r \\
& \leq C k^{3 / 2}\left(\sum_{j=0}^{n-1}\left\|u\left(t_{j}\right)\right\|_{\sigma+1}^{2 p}\right)^{\frac{1}{2 p}}\left(\sum_{j=0}^{n-1} \frac{2 p}{1^{2 p-1}}\right)^{\frac{2 p-1}{2 p}} \\
& \leq C k^{\frac{p+1}{2 p}}\left(\sum_{j=0}^{n-1}\left\|u\left(t_{j}\right)\right\|_{\sigma+1}^{2 p}\right)^{\frac{1}{2 p}}
\end{aligned}
$$

so that, by Proposition 5.1

$$
\begin{aligned}
\mathbb{E}\left[\max _{n=1, \ldots, N}\left\|I_{2}^{n}\right\|_{\sigma}^{2 p}\right] & \leq C k^{p+1} \sum_{j=0}^{N-1} \mathbb{E}\left[\left\|u\left(t_{j}\right)\right\|_{\sigma+1}^{2 p}\right] \\
& \leq C k^{p} .
\end{aligned}
$$

Again using Hölder's inequality, we have for $I_{3}^{n}$

$$
\begin{aligned}
\left\|I_{3}^{n}\right\|_{\sigma} & \leq \sum_{j=0}^{n-1} \int_{t_{j}}^{t_{j+1}}\left\|V(x)\left(u\left(t_{j}\right)-u^{j}\right)\right\|_{\sigma} \mathrm{d} r \\
& \leq C k \sum_{j=0}^{n-1}\|V(x)\|_{\sigma}\left\|u\left(t_{j}\right)-u^{j}\right\|_{\sigma} \\
& \leq C k^{\frac{1}{2 p}}\left(\sum_{j=0}^{n-1}\left\|u\left(t_{j}\right)-u^{j}\right\|_{\sigma}^{2 p}\right)^{\frac{1}{2 p}},
\end{aligned}
$$

and thus

$$
\mathbb{E}\left[\max _{n=1, \ldots, N}\left\|I_{3}^{n}\right\|_{\sigma}^{2 p}\right] \leq C k \sum_{j=0}^{N-1} \mathbb{E}\left[\max _{l=0, \ldots, j}\left\|u\left(t_{l}\right)-u^{l}\right\|_{\sigma}^{2 p}\right] .
$$

When estimating the stochastic integrals we will use the Burkholder inequality and that $[r / k]$ 
is the integer part of $r / k$. Using also Hölder's inequality, and Lemma 5.3, we have for $J_{1}^{n}$

$$
\begin{aligned}
\mathbb{E}\left[\max _{n=1, \ldots, N}\left\|J_{1}^{n}\right\|_{\sigma}^{2 p}\right] & \leq \mathbb{E}\left[\sup _{0 \leq t \leq T}\left\|\int_{0}^{t} S(t-[r / k] k)(u(r)-u([r / k] k)) \mathrm{d} W(r)\right\|_{\sigma}^{2 p}\right] \\
& \leq C \mathbb{E}\left[\left(\int_{0}^{T}\left\|(u(t)-u([t / k] k)) Q^{1 / 2}\right\|_{\mathscr{L}_{2}^{\sigma}}^{2} \mathrm{~d} t\right)^{p}\right] \\
& \leq C\left\|Q^{1 / 2}\right\|_{\mathscr{L}_{2}^{\sigma}}^{2 p} \mathbb{E}\left[\left(\left(\int_{0}^{T}\|u(t)-u([t / k] k)\|_{\sigma}^{2 p} \mathrm{~d} t\right)^{\frac{1}{p}} T^{\frac{p-1}{p}}\right)^{p}\right] \\
& \leq C \sum_{j=0}^{N-1} \int_{t_{j}}^{t_{j+1}} \mathbb{E}\left[\left\|u(t)-u\left(t_{j}\right)\right\|_{\sigma}^{2 p}\right] \mathrm{d} t \\
& \leq C \sum_{j=0}^{N-1} \int_{t_{j}}^{t_{j+1}}\left|t-t_{j}\right|^{p} \mathrm{~d} t \\
& \leq C k^{p} .
\end{aligned}
$$

Similarly for $J_{2}^{n}$, we have

$$
\begin{aligned}
\mathbb{E}\left[\max _{n=1, \ldots, N}\left\|J_{2}^{n}\right\|_{\sigma}^{2 p}\right] & \leq \mathbb{E}\left[\sup _{0 \leq t \leq T}\left\|\int_{0}^{t} S(t-[r / k] k)(S([r / k] k-r)-I) u([r / k] k) \mathrm{d} W(r)\right\|_{\sigma}^{2 p}\right] \\
& \leq C \mathbb{E}\left[\left(\int_{0}^{T}\left\|(S([t / k] k-t)-I) u([t / k] k) Q^{1 / 2}\right\|_{\mathscr{L}_{2}^{\sigma}}^{2} \mathrm{~d} t\right)^{p}\right] \\
& \leq C T^{p-1} \int_{0}^{T} \mathbb{E}\left[\left\|(S([t / k] k-t)-I) u([t / k] k) Q^{1 / 2}\right\|_{\left.\mathscr{L}_{2}^{\sigma}\right]}^{2 p}\right] \mathrm{d} t \\
& \leq C \sum_{j=0}^{N-1} \int_{t_{j}}^{t_{j+1}} \mathbb{E}\left[\| S\left(t_{j}-t\right)-I\right) u\left(t_{j}\right) Q^{1 / 2} \|_{\mathscr{L}_{2}^{\sigma}}^{2 p} \mathrm{~d} t \\
& \leq C\left\|Q^{1 / 2}\right\|_{\mathscr{L}_{2}^{\sigma+1}}^{2 p} \sum_{j=0}^{N-1} \int_{t_{j}}^{t_{j+1}}\left|t_{j}-t\right|^{p} \mathbb{E}\left[\left\|u\left(t_{j}\right)\right\|_{\sigma+1}^{2 p}\right] \mathrm{d} t \\
& \leq C k^{p} .
\end{aligned}
$$

For $J_{3}^{n}$, we have

$$
\begin{aligned}
\mathbb{E}\left[\max _{n=1, \ldots, N}\left\|J_{3}^{n}\right\|_{\sigma}^{2 p}\right] & \leq C \mathbb{E}\left[\sup _{0 \leq t \leq T}\left\|\int_{0}^{t} S(t-[r / k] k)\left(u([r / k] k)-u^{[r / k] k}\right) \mathrm{d} W(r)\right\|_{\sigma}^{2 p}\right] \\
& \leq C \mathbb{E}\left[\left(\int_{0}^{T}\left\|\left(u([t / k] k)-u^{[t / k] k}\right) Q^{1 / 2}\right\|_{\mathscr{L}_{2}^{\sigma}}^{2} \mathrm{~d} t\right)^{p}\right] \\
& \leq C T^{p-1}\left\|Q^{1 / 2}\right\|_{\mathscr{L}_{2}^{\sigma}}^{2 p} \sum_{j=0}^{N-1} \int_{t_{j}}^{t_{j+1}} \mathbb{E}\left[\left\|u\left(t_{j}\right)-u^{j}\right\|_{\sigma}^{2 p}\right] \mathrm{d} t \\
& \leq C k \sum_{j=0}^{N-1} \mathbb{E}\left[\max _{l=0, \ldots, j}\left\|u\left(t_{l}\right)-u^{l}\right\|_{\sigma}^{2 p}\right] .
\end{aligned}
$$

Putting all these estimates together yields

$$
\mathbb{E}\left[\max _{n=1, \ldots, N}\left\|u^{n}-u\left(t_{n}\right)\right\|_{\sigma}^{2 p}\right] \leq C_{1} k^{p}+C_{2} k \sum_{j=0}^{N-1} \mathbb{E}\left[\max _{l=0, \ldots, j}\left\|u^{l}-u\left(t_{l}\right)\right\|_{\sigma}^{2 p}\right],
$$

and Gronwall's lemma completes the proof. $\square$ 


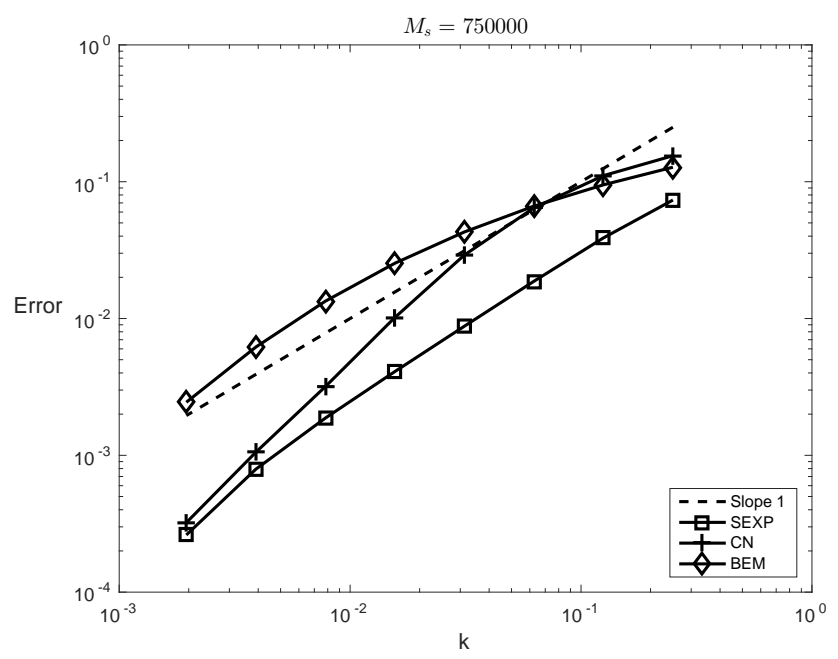

FIGURE 5.1. Stochastic Schrödinger equation with multiplicative noise: Mean-square errors for the stochastic exponential integrator (SEXP), the Crank-Nicolson scheme (CN), and the semi-implicit Euler-Maruyama (SEM). The dotted line has slope 1.

5.2. Numerical experiments for Schrödinger equations with a multiplicative noise. This subsection illustrates the convergence properties of the stochastic exponential integrator 5.2. when applied to the stochastic partial differential equation (5.1) on the interval $[0,2 \pi]$ with periodic boundary conditions. In these numerical experiments, we set $V(x)=0$ and $u_{0}(x)=\mathrm{e}^{-5(x-\pi)^{2}}$. We compare the stochastic exponential integrator (SEXP) with the CrankNicolson scheme $(\mathrm{CN})$ and the semi-implicit Euler-Maruyama scheme (SEM).

Figure 5.1 illustrates the convergence errors of the above numerical methods for a noise with covariance operator having the eigenvalues $\lambda_{n}=1 /\left(1+|n|^{5.1}\right)$ for $n \in \mathbb{Z}$. The spatial discretisation is done by a pseudospectral method with $M=2^{8}$ Fourier modes. The rates of mean-square convergence (measured in the $L^{2}$-norm at the end of the interval of integration $[0,0.5])$ of these numerical methods are presented in this figure. The expected rate of convergence $\mathscr{O}\left(k^{1}\right)$ of the stochastic exponential integrator, as stated in Theorem 5.5 can be confirmed. Here, the exact solution is approximated by the stochastic exponential method with a very small time step $k_{\text {exact }}=2^{-10}$ and $M_{\mathrm{s}}=750000$ samples are used for the approximation of the expected values.

\section{REFERENCES}

[1] R. Anton, D. Cohen, S. Larsson, And X. WAng, Full discretisation of semi-linear stochastic wave equations driven by multiplicative noise, Submitted for publication, (2015).

[2] M. Barton-Smith, A. Debussche, And L. Di Menza, Numerical study of two-dimensional stochastic NLS equations, Numer. Methods Partial Differential Equations, 21 (2005), pp. 810-842.

[3] H. BERLAND, A. L. ISLAS, AND C. M. SCHOBER, Conservation of phase space properties using exponential integrators on the cubic Schrödinger equation, J. Comput. Phys., 225 (2007), pp. 284-299.

[4] H. Berland, B. OWren, AND B. SKAFLESTAD, Solving the nonlinear Schrãüdinger equation using exponential integrators, Modeling, Identification and Control, 27 (2006), pp. 201-218.

[5] B. CANO AND A. GONZÁLEZ-PACHÓN, Exponential time integration of solitary waves of cubic Schrödinger equation, Appl. Numer. Math., 91 (2015), pp. 26-45.

[6] T. Cazenave and A. Haraux, An Introduction to Semilinear Evolution Equations, vol. 13 of Oxford Lecture Series in Mathematics and its Applications, The Clarendon Press, Oxford University Press, New York, 1998. Translated from the 1990 French original by Yvan Martel and revised by the authors. 
[7] E. Celledoni, D. Cohen, AND B. OWren, Symmetric exponential integrators with an application to the cubic SchrÂúdinger equation, Found. Comput. Math., 8 (2008), pp. 303-317.

[8] D. COHEN AND L. GAUCKLER, Exponential integrators for nonlinear SchrÃüdinger equations over long times, BIT, 52 (2012), pp. 877-903.

[9] D. Cohen, S. LARSSON, AND M. SigG, A trigonometric method for the linear stochastic wave equation, SIAM J. Numer. Anal., 51 (2013), pp. 204-222.

[10] D. COHEN AND L. QUER-SARDANYONS, A fully discrete approximation of the one-dimensional stochastic wave equation, IMA Journal of Numerical Analysis, (2015). http://dx.doi.org/10.1093/imanum/drv006.

[11] G. Da Prato And J. ZabczYK, Stochastic Equations in Infinite Dimensions, vol. 44 of Encyclopedia of Mathematics and its Applications, Cambridge University Press, Cambridge, 1992.

[12] A. DE BOUARD AND A. DEBUSSCHE, A stochastic nonlinear Schrödinger equation with multiplicative noise, Comm. Math. Phys., 205 (1999), pp. 161-181.

[13] - On the effect of a noise on the solutions of the focusing supercritical nonlinear Schrödinger equation, Probab. Theory Related Fields, 123 (2002), pp. 76-96.

[14] — The stochastic nonlinear Schrödinger equation in $H^{1}$, Stochastic Anal. Appl., 21 (2003), pp. 97-126.

[15] A. De Bouard AND A. Debussche, A semi-discrete scheme for the stochastic nonlinear Schrödinger equation, Numer. Math., 96 (2004), pp. 733-770.

[16] A. DE BouARD AND A. DeBussche, Blow-up for the stochastic nonlinear Schrödinger equation with multiplicative noise, Ann. Probab., 33 (2005), pp. 1078-1110.

[17] - Weak and strong order of convergence of a semidiscrete scheme for the stochastic nonlinear Schrödinger equation, Appl. Math. Optim., 54 (2006), pp. 369-399.

[18] A. De Bound, A. Debussche, And L. Di Menza, Theoretical and numerical aspects of stochastic nonlinear Schrödinger equations, Monte Carlo Methods Appl., 7 (2001), pp. 55-63. Monte Carlo and probabilistic methods for partial differential equations (Monte Carlo, 2000).

[19] A. Debussche And L. Di MenZa, Numerical simulation of focusing stochastic nonlinear Schrödinger equations, Phys. D, 162 (2002), pp. 131-154.

[20] G. Dujardin, Exponential Runge-Kutta methods for the Schrödinger equation, Appl. Numer. Math., 59 (2009), pp. 1839-1857.

[21] E. Hebey, Nonlinear Analysis on Manifolds: Sobolev Spaces and Inequalities, vol. 5 of Courant Lecture Notes in Mathematics, New York University, Courant Institute of Mathematical Sciences, New York; American Mathematical Society, Providence, RI, 1999.

[22] M. HochBRUCK AND C. LUBICH, Exponential integrators for quantum-classical molecular dynamics, BIT, 39 (1999), pp. 620-645.

[23] M. Hochbruck AND A. Ostermann, Exponential integrators, Acta Numer., 19 (2010), pp. 209-286.

[24] A. JentZEN AND P. E. KloEden, The numerical approximation of stochastic partial differential equations, Milan J. Math., 77 (2009), pp. 205-244.

[25] S. JiAng, L. WANG, AND J. Hong, Stochastic multi-symplectic integrator for stochastic nonlinear Schrödinger equation, Commun. Comput. Phys., 14 (2013), pp. 393-411.

[26] J. LiU, A mass-preserving splitting scheme for the stochastic Schrödinger equation with multiplicative noise, IMA J. Numer. Anal., 33 (2013), pp. 1469-1479.

[27] — Order of convergence of splitting schemes for both deterministic and stochastic nonlinear Schrödinger equations, SIAM J. Numer. Anal., 51 (2013), pp. 1911-1932.

[28] G. J. LORD AND J. Rougemont, A numerical scheme for stochastic PDEs with Gevrey regularity, IMA J. Numer. Anal., 24 (2004), pp. 587-604.

[29] G. J. Lord AND A. TAmbUe, Stochastic exponential integrators for the finite element discretization of SPDEs for multiplicative and additive noise, IMA J. Numer. Anal., 33 (2013), pp. 515-543.

[30] C. M. Mora AND R. ReBolledo, Regularity of solutions to linear stochastic Schrödinger equations, Infin. Dimens. Anal. Quantum Probab. Relat. Top., 10 (2007), pp. 237-259.

[31] A. H. Strømmen Melb $\emptyset$ And D. J. Higham, Numerical simulation of a linear stochastic oscillator with additive noise, Appl. Numer. Math., 51 (2004), pp. 89-99.

[32] X. WANG, An exponential integrator scheme for time discretization of nonlinear stochastic wave equation, Journal of Scientific Computing, (2014), pp. 1-30. 